\title{
Quantum Deformation of Lorentz Group
}

\author{
P. Podleś and S. L. Woronowicz \\ Department of Mathematical Methods in Physics, Faculty of Physics, \\ University of Warsaw, Hoza 74, PL-00-682 Warszawa, Poland
}

\begin{abstract}
A one parameter quantum deformation $S_{\mu} L(2, \mathbb{C})$ of $S L(2, \mathbb{C})$ is introduced and investigated. An analog of the Iwasawa decomposition is proved. The compact part of this decomposition coincides with $S_{\mu} U(2)$, whereas the solvable part is identified as a Pontryagin dual of $S_{\mu} U(2)$. It shows that $S_{\mu} L(2, \mathbb{C})$ is the result of the dual version of Drinfeld's double group construction applied to $S_{\mu} U(2)$. The same construction applied to any compact quantum group $G_{c}$ is discussed in detail. In particular the explicit formulae for the Haar measures on the Pontryagin dual $G_{d}$ of $G_{c}$ and on the double group $G$ are given. We show that there exists remarkable $1-1$ correspondence between representations of $G$ and bicovariant bimodules ("tensor bundles") over $G_{c}$. The theory of smooth representations of $S_{\mu} L(2, \mathbb{C})$ is the same as that of $S L(2, \mathbb{C})$ (Clebsh-Gordon coefficients are however modified). The corresponding "tame" bicovariant bimodules on $S_{\mu} U(2)$ are classified. An application to $4 D_{+}$differential calculus is presented. The nonsmooth case is also discussed.
\end{abstract}

\section{Introduction}

Despite 60 years of intensive efforts of many eminent physicists quantum theory is not yet fully compatible with the (special) theory of relativity. The failure of the program of constructive quantum field theory is one of many manifestations of this incompatibility. More detailed analysis shows that the difficulty lies in small space distances. It seems that the four-dimensional smooth pseudoriemannian manifold is a good model of our space-time only in the macro-scale. The description of the space-time in sub-micro level may require the new tools provided by a noncommutative generalization of differential geometry $[2,9,12]$.

It is not clear what the symmetry properties of the space-time in the sub-micro scale are. The idea that the symmetry properties are described by a quantum group $[3,8,10]$ is very attractive. By virtue of the correspondence principle the symmetry group should be a deformation of the Poincaré group.

The Poincare group is the semidirect product of Lorentz and translation groups. Therefore at the first step we should construct a quantum deformation of 
the Lorentz group. The present paper completely solves this problem. We introduce a one parameter family of quantum groups. All relevant notions such as comultiplication, counit, coinverse and the Haar measure are introduced and discussed in detail. Finite-dimensional representations are investigated. For a special value of the parameter one obtains the classical Lorentz group.

Throughout the paper by (quantum) Lorentz group we mean the (quantum) $S L(2, \mathbb{C})$ group. Only in the last section we briefly discuss the quantum analog of the $S O(3,1)$ group. In many places we refer to the terminology introduced in $[8]$, replacing however the word "pseudo" by the recently more fashionable term "quantum".

The paper is organized in the following way. In Sect. 1 we discuss the commutation relations defining the algebra of continuous functions on the quantum Lorentz group. These relations are imposed on matrix elements of the fundamental representation acting on the two-dimensional space of "undotted spinors". To derive the relations we shall assume that the fundamental representation obeys the usual properties known in the theory of spinors and that the quantum Lorentz group contains $S_{\mu} U(2)$ (where $\mu \in[-1,1] \backslash\{0\}$ is a fixed parameter) in the same way as $S L(2, \mathbb{C})$ contains $S U(2)$.

The Iwasawa decomposition theorem (Theorem 1.3) is the main result of Sect. 1. It shows that the quantum Lorentz group is in a certain sense the product of two subgroups. The first one is the $S_{\mu} U(2)$ subgroup mentioned above. The second "solvable subgroup" is (as we shall see in Sect. 5) isomorphic to the Pontryagin dual of $S_{\mu} U(2)$.

It turns out that for any compact quantum group $G_{c}$ there exists a quantum group $G$ that contains $G_{c}$ and the Pontryagin dual of $G_{c}$ combined in a canonical nontrivial way. $G$ is called the double group built over $G_{c}$.

The construction of the double group is carried out in Sect. 4. Sections 2 and 3 contain necessary preparatory material. In Sect. 2 we introduce the convolution product and the Fourier transform of "continuous functions" on a compact quantum (matrix) group $G_{c}$. The quantum space $G_{d}$ of all characters on $G_{c}$ is found. Section 2 may be regarded as a supplement to [10]. Section 3 is devoted to the Pontryagin duals. By definition (cf. [8]) the Pontryagin dual of $G_{c}$ is the quantum space $G_{d}$ endowed with the natural group structure. We study in detail the comultiplication, counit, coinverse and Haar measures related to $G_{d}$. We show that $G_{d}$ is unimodular if and only if the Haar measure on $G_{c}$ is central. The duality between $G_{c}$ and $G_{d}$ is described by a unitary bicharacter $u$. At the end of Sect. 3 finitedimensional representations of $G_{d}$ are investigated. It turns out that there exists natural 1-1 correspondence between the set of all representations of $G_{d}$ acting on a finite-dimensional vector space $K$ and the set of all homomorphisms of the algebra of smooth functions of $G_{c}$ into $B(K)$. In Sect. 4 we use the dual version of Drinfeld's construction [3] in order to introduce the double group $G$ built over $G_{c}$. As a quantum space $G$ is homeomorphic to the cartesian product $G_{c} \times G_{d}$. $G$ contains $G_{c}$ and $G_{d}$ as subgroups. However the quantum group $G$ is not a direct product of $G_{c}$ and $G_{d}$. The point is that inside $G$ the "elements" of $G_{c}$ do not commute with "elements" of $G_{d}$. This noncommutativity is described by a homeomorphism $\sigma_{*}: G_{d} \times G_{c} \rightarrow G_{c} \times G_{d}$ defined by (4.9). Using this homeomorphism we introduce the group structure (the comultiplication, counit and coinverse) 
on $G$. It turns out that there exists on $G$ a left and right invariant Haar measure ( $G$ is unimodular!).

A large part of Sect. 4 is devoted to finite-dimensional representations of the double group. Restricting a representation $\pi$ of $G$ to $G_{c}\left(G_{d}\right.$ respectively) we obtain a representation $\pi_{c}$ of $G_{c}\left(\pi_{d}\right.$ of $G_{d}$ respectively). We show that $\pi$ is uniquely determined by the pair $\left(\pi_{c}, \pi_{d}\right)$ and that $\pi_{c}$ and $\pi_{d}$ satisfy a certain compatibility condition. Conversely any pair $\left(\pi_{c}, \pi_{d}\right)$ satisfying the compatibility condition gives rise to a representation of $G$. The compatibility condition turns out to be equivalent to the fundamental formula of the theory of bicovariant bimodules (formula (2.39) of [12]). Due to this fact there exists one to one natural correspondence between representations of $G$ and bicovariant bimodules over the algebra of smooth functions on $G_{c}$.

In Sect. 5 the general theory developed in Sects. 2, 3, and 4 is applied to the group $G_{c}=S_{\mu} U(2)$. It turns out that in this case the double group obeys all the properties considered in Sect. 1. This way the construction of the quantum Lorentz group is completed. Section 6 is devoted to the study of finite-dimensional representations of the quantum Lorentz group. To our surprise we discover the existence of non-smooth representations. At the end of this section we classify the bicovariant bimodules over $S_{\mu} U(2)$. An independent description of the $4 D_{+}-$ differential calculus is given.

Many quantum groups that we deal with in this paper are not compact. The non-compactness causes the difficulties of a conceptual and technical nature. The first problem is related to the fact that no general theory of non-compact quantum groups exists. In particular no precise definition of non-compact quantum group is yet formulated. In our opinion it is too early to formulate the general theory of quantum groups in an axiomatic way (as was done for the compact case in [10]). At first we have to elaborate a number of examples in order to collect the necessary experience in the subject. We believe that the present paper makes an important step in this program.

Technically non-compactness means that we work with non-unital $C^{*}$-algebras. Let us remind (cf. [8]) that in the category of such algebras a morphism $\phi \in \operatorname{Mor}(A, B)$ is by definition a linear, multiplicative, *-preserving mapping from $A$ into $M(B)$ such that $\phi(A) B$ is dense in $B$. In this definition and in the rest of the paper $M(A)$ denotes the multiplier algebra of a $C^{*}$-algebra $A$. In particular if $A$ is the algebra of "all vanishing at infinity continuous functions" on a non-compact quantum group then the comultiplication $\Phi \in \operatorname{Mor}(A, A \otimes A)$ is an *-algebra homomorphism acting from $A$ into $M(A \otimes A)$. Since in general $M(A) \otimes M(A) \varsubsetneqq M(A \otimes A)$, the Hopf-algebra formalism does not work in noncompact case.

The second difficulty produced by the non-compactness is related to the necessity of working with unbounded elements affiliated with $C^{*}$-algebras. According to [13], $a$ is an element affiliated with a $C^{*}$-algebra $A$ if $a$ is an unbounded (satisfying certain conditions) multiplier densely defined on $A$. In this case we write $a \eta A$. Let $A$ be the $C^{*}$-algebra of "all continuous vanishing at infinity functions" on a non-compact quantum group $G$ and $a$ be a matrix element of a finite-dimensional representation of $G$. Then in general $a$ is unbounded and the relation $a \in A$ can not hold. Instead of this relation we require that $a \eta A$. 
Let $A$ be a $C^{*}$-algebra. Elements of $M(A)$ are the only bounded elements affiliated with $A$. If $A$ is unital then $a \eta A$ iff $a \in A$. Elements affiliated with a $C^{*}$-algebra can be transported by morphisms: if $a \eta A$ and $\phi \in \operatorname{Mor}(A, B)$ then $\phi(a)$ is a well defined element affiliated with $B$.

All non-unital $C^{*}$-algebras considered in this paper are of the form

$$
A=\sum_{n \in N}^{\oplus} A_{n},
$$

where $N$ is a denumerable set and $A_{n}$ are $C^{*}$-algebras with unity. Any element $a \eta A$ is of the form

$$
a=\sum_{n \in N}^{\oplus} a_{n},
$$

where $a_{n} \in A_{n}$ for any $n$. If $\lim _{n}\left\|a_{n}\right\|=0\left(\sup _{n}\left\|a_{n}\right\|<\infty\right.$ respectively) then $a \in A$ see Remark on attached sheet of paper $(a \in M(A)$ respectively). It is clear that the set of all elements affiliated with $A$ is endowed with the natural *-algebra structure (it is a pro- $C^{*}$-algebra, cf. [6]). One can easily verify that the Pedersen ideal $A_{0}$ of $A$ is the set of all elements of the form (0.1) where $a_{n}=0$ for all except a finite number of $n$. Let

$$
B=\sum_{m \in M}^{\oplus} B_{m}
$$

be another $C^{*}$-algebra of considered form and

$$
\phi: A_{0} \ni a \rightarrow \phi(a) \eta B
$$

be a linear mapping. We say that $\phi$ is proper if for any $m \in M$ there exists a finite subset $N_{m} \subseteq N$ such that for all $a \in A_{0}$,

$$
\left(\begin{array}{c}
a_{n}=0 \\
\text { for all } n \in N_{m}
\end{array}\right) \Rightarrow\left(\phi(a)_{m}=0\right) \text {. }
$$

One can easily check that any $\phi \in \operatorname{Mor}(A, B)$ is proper. If $\phi$ is proper then $\phi(a)_{m}$ depends only on a finite number of components of $a$. Therefore $\phi$ admits a natural extension to the set of all elements affiliated with $A$. We shall also use the notion of proper linear mapping in a more complicated setting. In some cases $\phi$ is defined only on a dense *-subalgebra $\mathscr{A} C A$ of the form

$$
\mathscr{A}=\sum_{\text {fin }}^{\oplus} \mathscr{A}_{n},
$$

where for each $n, \mathscr{A}_{n}$ is a dense unital $*$-subalgebra of $A_{n}$. By definition

$$
\sum_{\text {fin }}^{\oplus} \mathscr{A}_{n}=\left\{a \in A_{0}: a_{n} \in \mathscr{A}_{n} \text { for any } n\right\} .
$$

If $\phi$ is proper then $\phi$ admits a natural extension to the set of all elements a $A$ such that $a_{n} \in \mathscr{A}_{n}$ for all $n$.

Let

$$
A=\sum^{\oplus} A_{n}, \quad A^{\prime}=\sum^{\oplus} A_{k}^{\prime}
$$


be $C^{*}$-algebras of considered form and

$$
\begin{aligned}
& \mathscr{A}=\sum_{\mathrm{fin}}^{\oplus} \mathscr{A}_{n} \subset A, \\
& \mathscr{A}^{\prime}=\sum_{\text {fin }}^{\oplus} \mathscr{A}_{k}^{\prime} \subset A^{\prime}
\end{aligned}
$$

be dense *-subalgebras. We set

$$
\mathscr{A} \otimes_{r} \mathscr{A}^{\prime} \stackrel{\mathrm{df}}{=}\left\{a \eta A \otimes A^{\prime}: a_{n k} \in \mathscr{A}_{n} \otimes_{a l g} \mathscr{A}_{k}^{\prime} \text { for all } n, k\right\} .
$$

If $B$ and $B^{\prime}$ are $C^{*}$-algebras of considered form and

$$
\begin{gathered}
\phi: \mathscr{A} \rightarrow B, \\
\phi^{\prime}: \mathscr{A}^{\prime} \rightarrow B^{\prime},
\end{gathered}
$$

are proper linear mappings, then $\phi \otimes \phi^{\prime}$ is proper and admits a natural extension to $\mathscr{A} \otimes_{r} \mathscr{A}^{\prime}$. In what follows, dealing with proper linear mappings we shall freely use their natural extensions without any further comment.

In the paper we constantly consider tensor products of $C^{*}$-algebras. Dealing with elements of such tensor products it is in some cases convenient to use "the leg numbering notation". We shall explain this notation in the simplest possible case:

Let $A, B$ and $C$ be $C^{*}$-algebras and $\phi_{12} \in \operatorname{Mor}(A \otimes B, A \otimes B \otimes C)$, $\phi_{13} \in \operatorname{Mor}(A \otimes C, A \otimes B \otimes C)$ and $\phi_{23} \in \operatorname{Mor}(B \otimes C, A \otimes B \otimes C)$ be canonical embeddings, i.e.

$$
\begin{aligned}
& \phi_{12}(x \otimes y)=x \otimes y \otimes I_{C}, \\
& \phi_{13}(x \otimes z)=x \otimes I_{B} \otimes z, \\
& \phi_{23}(y \otimes z)=I_{A} \otimes y \otimes z,
\end{aligned}
$$

for any $x \in A, y \in B$, and $z \in C\left(I_{A}\left(I_{B}\right.\right.$ and $I_{C}$ respectively) denotes the unity of $M(A)$ $(M(B)$ and $M(C)$ respectively)). For any $c \eta A \otimes B, b \eta A \otimes C$, and $a \eta B \otimes C$ we set

$$
\begin{aligned}
& c_{12}=\phi_{12}(c), \\
& b_{13}=\phi_{13}(b), \\
& a_{23}=\phi_{23}(a) .
\end{aligned}
$$

Clearly $c_{12}, b_{13}$, and $a_{23}$ are affiliated with $A \otimes B \otimes C$. The reader should notice some ambiguity hidden in this notation. If $b \eta A \otimes C$ then $b_{13}$ is the corresponding element affiliated with $A \otimes B \otimes C$, where $B$ is a $C^{*}$-algebra that is determined by the context.

We shall also use the $\odot$ and $(1)$ products introduced in $[10]$. If $u \eta B(K) \otimes A$ and $v \eta B(K) \otimes B$ (where $K$ is a finite-dimensional vector space and $A, B$ are $C^{*}$-algebras) then

$$
u\left(\stackrel{)}{\stackrel{\mathrm{df}}{=}} u_{12} v_{13} .\right.
$$


Clearly $u \bowtie v \eta B(K) \otimes A \otimes B$. Similarly if $u \eta B(K) \otimes A$ and $w \eta B(L) \otimes A$ (where $K, L$ are finite-dimensional vector spaces and $A$ is a $C^{*}$-algebra) then

Clearly $u \Theta w \eta B(K \otimes L) \otimes A$.

$$
u \odot w \stackrel{\mathrm{df}}{=} u_{13} w_{23} \text {. }
$$

Dealing with linear mappings acting between tensor products of $C^{*}$-algebras one may use the diagram notation that generalizes that introduced in [11] (see Appendix A for details).

\section{First Encounter with Quantum Lorentz Group}

Introducing a quantum group one has first to define the algebra of "continuous functions" on it. In most cases this algebra is introduced in terms of generators and commutation relations. Usually the generators are matrix elements of the fundamental representation of the group and the relations reflect (specific for each considered quantum group) desired properties of this representation. For example in the case of $S_{\mu} U(2)$ the commutation relations mean that the fundamental representation is unitary and irreducible and that the tensor square of this representation contains the trivial representation.

We follow the same methodology introducing the quantum Lorentz group $S_{\mu} L(2, \mathbb{C}), \mu \in[-1,1] \backslash\{0\}$. We start with the two-dimensional fundamental representation

$$
w=\left(\begin{array}{ll}
\alpha, & \beta \\
\gamma, & \delta
\end{array}\right)
$$

corresponding to the representation of classical Lorentz group acting on the space of "undotted" spinors. Copying the classical case we shall assume that the tensor square of $w$ is equivalent to $w^{0} \oplus w^{1}$, where $w^{0}$ is one-dimensional trivial representation and $\operatorname{dim} w^{1}=3$. It means that there exist $E \in B\left(\mathbb{C}^{1}, \mathbb{C}^{2} \otimes \mathbb{C}^{2}\right)$ and $E^{\prime} \in B\left(\mathbb{C}^{2} \otimes \mathbb{C}^{2}, \mathbb{C}^{1}\right)$ such that

$$
\begin{gathered}
(w \circledast w)(E \otimes I)=E \otimes I, \\
\left(E^{\prime} \otimes I\right)(w \circledast w)=E^{\prime} \otimes I,
\end{gathered}
$$

and $E^{\prime} E \neq 0(E(1)$ is the ( $w \odot w)$-invariant vector generating the subspace related to $w^{0} ; E^{\prime}$ is the $(w \oplus w)$-invariant functional killing the three-dimensional subspace related to $\left.w^{1}\right)$.

Let $\bar{w}$ be the complex conjugate of $w$ :

$$
\bar{w}=w^{j \otimes *},
$$

where $j$ denotes an antilinear, multiplicative invertible mapping acting on the algebra of all complex $2 \times 2$ matrices: $M_{2}(\mathbb{C}) \ni m \rightarrow m^{j} \in M_{2}(\mathbb{C})$. The representation $\bar{w}$ corresponds to the representation of the classical Lorentz group acting on the space of "dotted" spinors. Having in mind the classical case we shall assume that $\bar{w} \odot w$ is irreducible and that $w \odot \bar{w}$ is equivalent to $\bar{w} \odot w$. The latter means that there exists an invertible linear mapping $\lambda \in B\left(\mathbb{C}^{2} \otimes \mathbb{C}^{2}\right)$ such that

$$
(\lambda \otimes I)(\bar{w} \odot w)=(w \odot \bar{w})(\lambda \otimes I) .
$$


In order to specify $E, E^{\prime}, j, \lambda$ entering formulae (1.2)-(1.5) we shall assume that $S_{\mu} L(2, \mathbb{C})$ contains $S_{\mu} U(2)$ as a subgroup and that $w$ and $\bar{w}$ restricted to $S_{\mu} U(2)$ coincide with the fundamental representation of $S_{\mu} U(2)$. This representation will be denoted by $w_{c}$. It means that relations (1.2)-(1.5) must be satisfied if we replace $w$ and $\bar{w}$ by $w_{c}$.

Let $\left(\varepsilon_{1}, \varepsilon_{2}\right)$ be the canonical basis in $\mathbb{C}^{2}$. Using the known properties of the fundamental representation of $S_{\mu} U(2)$ (cf. [9]) we see that

$$
\left(\begin{array}{ll}
z_{1}, & z_{2} \\
z_{3}, & z_{4}
\end{array}\right)^{j}=\left(\begin{array}{cc}
\bar{z}_{4}, & -\mu \bar{z}_{3} \\
-\mu^{-1} \bar{z}_{2}, & \bar{z}_{1}
\end{array}\right)
$$

for any $z_{1}, z_{2}, z_{3}, z_{4} \in \mathbb{C}$ and up to a numerical factor,

$$
\left.\begin{array}{rl}
E(1) & =\varepsilon_{1} \otimes \varepsilon_{2}-\mu \varepsilon_{2} \otimes \varepsilon_{1} \\
E^{\prime}=E^{*} & =\text { hermitian conjugate of } E \\
\lambda & =\operatorname{id}_{\mathbb{C}^{2} \otimes \mathbb{C}^{2}}+s E E^{*}
\end{array}\right\},
$$

where $s \in \mathbb{C}$. In order to fix the value of $s$ we notice that $G=\left(E^{*} \otimes \mathrm{id} \otimes \mathrm{id}\right)$ $(\mathrm{id} \otimes \lambda \otimes \mathrm{id})(\lambda \otimes E)$ intertwines $\bar{w} \odot w$ with itself. Therefore (we want $\bar{w} \odot w$ to be irreducible) $G$ is a multiple of identity,

$$
\left(\varepsilon_{2} \otimes \varepsilon_{1} \mid G \varepsilon_{1} \otimes \varepsilon_{2}\right)=-\mu\left(\mu^{2} s^{2}+\left(1+\mu^{2}\right) s+1\right)=0
$$

and $s=-\mu^{-2}$ (s=-1 leads to an equivalent theory).

We shall write relations (1.1)-(1.5) in an expanded form. By virtue of (1.4), (1.1), and (1.6),

$$
\bar{w}=\left(\begin{array}{cc}
\delta^{*}, & -\mu \gamma^{*} \\
-\mu^{-1} \beta^{*}, & \alpha^{*}
\end{array}\right)
$$

and taking into account (1.7) one can easily verify that (1.1)-(1.5) are equivalent to the following set of relations:

$$
\begin{gathered}
\alpha \beta=\mu \beta \alpha, \\
\alpha \gamma=\mu \gamma \alpha, \\
\alpha \delta-\mu \beta \gamma=I, \\
\beta \gamma=\gamma \beta, \\
\beta \delta=\mu \delta \beta, \\
\gamma \delta=\mu \delta \gamma, \\
\delta \alpha-\mu^{-1} \beta \gamma=I, \\
\beta \alpha^{*}=\mu^{-1} \alpha^{*} \beta+\mu^{-1}\left(1-\mu^{2}\right) \gamma^{*} \delta, \\
\gamma \alpha^{*}=\mu \alpha^{*} \gamma, \\
\delta \alpha^{*}=\alpha^{*} \delta, \\
\gamma \beta^{*}=\beta^{*} \gamma, \\
\delta \beta^{*}=\mu \beta^{*} \delta-\mu\left(1-\mu^{2}\right) \alpha^{*} \gamma,
\end{gathered}
$$




$$
\begin{gathered}
\delta \gamma^{*}=\mu^{-1} \gamma^{*} \delta, \\
\alpha \alpha^{*}=\alpha^{*} \alpha+\left(1-\mu^{2}\right) \gamma^{*} \gamma, \\
\beta \beta^{*}=\beta^{*} \beta+\left(1-\mu^{2}\right)\left[\delta^{*} \delta-\alpha^{*} \alpha\right]-\left(1-\mu^{2}\right)^{2} \gamma^{*} \gamma, \\
\gamma \gamma^{*}=\gamma^{*} \gamma, \\
\delta \delta^{*}=\delta^{*} \delta-\left(1-\mu^{2}\right) \gamma^{*} \gamma .
\end{gathered}
$$

This way we have found the complete list of relations defining the algebra of continuous functions on the quantum Lorentz group. The construction of algebra itself is however much more complicated than in the compact group case (generators $\alpha, \beta, \gamma, \delta$ are unbounded, whereas we are interested in the $C^{*}$-algebra of "continuous, vanishing at infinity functions"). This construction is carried out in the forthcoming sections where also comultiplication, counit, coinverse and Haar measure will be considered.

In the remaining part of this section we investigate formal properties of the set of relations (1.9)-(1.25). In particular we discover a remarkable version of the Iwasawa decomposition.

In many formulae in this paper, the square root of $\mu$ is involved. In order to avoid the double meaning possible in the case $\mu<0$ we shall use the following convention:

If $\mu<0$, then for any $x \in \mathbb{R}$,

$$
\mu^{x}=|\mu|^{x} e^{i \pi x} .
$$

Moreover we set

$$
S_{\mu}=\left\{\mu^{x}: x \in \mathbb{R}\right\} \cup\{0\}
$$

If $\mu>0$ then $S_{\mu}=\overline{\mathbb{R}}_{+}$.

It is well known that the group $G=S L(2, \mathbb{C})$ admits the following decomposition: $G=K \cdot A N$, where $K$ and $A N$ are subgroups of $G$ :

$$
\begin{gathered}
K=S U(2)=\{u \in G: u-\text { unitary }\}, \\
A N=\left\{\left(\begin{array}{cc}
a, & n \\
0, & a^{-1}
\end{array}\right): a \in \mathbb{R}, a>0, n \in \mathbb{C}\right\} .
\end{gathered}
$$

We shall show that a similar result holds for the quantum Lorentz group.

Remark. In the classical case the solvable subgroup admits the further decomposition: $A N=A \cdot N$, where $A$ is abelian and $N$ is a nilpotent subgroup of $A N$. However we were not able to find any analog of this decomposition in the quantum case.

Let $H$ be a Hilbert space and $M_{2}(B(H))$ be the set of all $2 \times 2$ matrices with entries belonging to $B(H)$. An element $w \in M_{2}(B(H))$ is said to be an $S_{\mu} L(2, \mathbb{C})$ matrix if the matrix elements of $w$

$$
w=\left(\begin{array}{ll}
\alpha, & \beta \\
\gamma, & \delta
\end{array}\right)
$$


satisfy the relations (1.9)-(1.25). If in addition $w$ is unitary then we say that $w$ is a $K_{\mu}$ (or $S_{\mu} U(2)$ )-matrix). A matrix $w_{c}$ is an $S_{\mu} U(2)$-matrix if and only if

$$
w_{c}=\left(\begin{array}{cc}
\alpha_{c}, & -\mu \gamma_{c}^{*} \\
\gamma_{c}, & \alpha_{c}^{*}
\end{array}\right),
$$

where $\alpha_{c}, \gamma_{c} \in B(H)$ satisfy the relations

$$
\begin{gathered}
\alpha_{c}^{*} \alpha_{c}+\gamma_{c}^{*} \gamma_{c}=I, \\
\alpha_{c} \alpha_{c}^{*}+\mu^{2} \gamma_{c}^{*} \gamma_{c}=I, \\
\gamma_{c}^{*} \gamma_{c}=\gamma_{c} \gamma_{c}^{*}, \\
\mu \gamma_{c} \alpha_{c}=\alpha_{c} \gamma_{c}, \\
\mu \gamma_{c}^{*} \alpha_{c}=\alpha_{c} \gamma_{c}^{*} .
\end{gathered}
$$

If (1.28) is unitary then these relations are automatically satisfied.

Let $w_{d} \in M_{2}(B(H))$ be a matrix with the left-lower-corner element equal to zero. One can easily check that $w_{d}$ is an $S_{\mu} L(2, \mathbb{C})$-matrix if and only if

$$
w_{d}=\left(\begin{array}{cc}
a, & n \\
0, & a^{-1}
\end{array}\right),
$$

where $a, n \in B(H)$ satisfy relations

$$
\begin{gathered}
a a^{*}=a^{*} a, \\
a n=\mu n a, \\
n a^{*}=\mu^{-1} a^{*} n, \\
n n^{*}=n^{*} n+\left(1-\mu^{2}\right)\left(\left(a^{*} a\right)^{-1}-a^{*} a\right) .
\end{gathered}
$$

If in addition $S p a \subset S_{\mu}$ (this condition replaces the inequality " $a>0$ " in (1.26)) then we say that $w_{d}$ is an $A N_{\mu}$-matrix.

Remark. According to Manin a quantum group is just the set of all matrices whose matrix elements satisfy assumed commutation relations [5]. E. g. $S_{\mu} U(2)$ is simply the set of all $S_{\mu} U(2)$-matrices. In our opinion this point of view, hardly accepted in the compact case, is at the moment completely useless in the theory of noncompact quantum groups (the theory presented in [4] is not yet generalized for non-unital $C^{*}$-algebras).

In the following propositions $G$ is one of the following three symbols: $S_{\mu} L(2, \mathbb{C})$, $S_{\mu} U(2), A N_{\mu}$. Moreover we say that two operators $p, q \in B(H)$ doubly commute if $p q=q p$ and $p q^{*}=q^{*} p$.

Proposition 1.1. Let $w^{1}$ and $w^{2}$ be G-matrices. Assume that matrix elements of $w^{1}$ doubly commute with matrix elements of $w^{2}$. Then $w^{1} w^{2}$ is a G-matrix.

Proof. The case $G=S_{\mu} L(2, \mathbb{C})$ has to be verified by direct computations. The computations become trivial if one remembers that (1.9)-(1.25) are equivalent to $(1.2)-(1.5)$. We know that the product of unitaries is unitary and the $S_{\mu} U(2)$-case follows. The same is true for $A N_{\mu}$-case (the product of triangular matrices is a triangular matrix and $t_{1} t_{2} \in S_{\mu}$ for $\left.t_{1}, t_{2} \in S_{\mu}\right)$. Q.E.D. 
As a simple conclusion we get immediately

Proposition 1.2. Let $H^{1}$ and $H^{2}$ be Hilbert spaces and $w^{1} \in M_{2}\left(B\left(H^{1}\right)\right)$, $w^{2} \in M_{2}\left(B\left(H^{2}\right)\right)$. Assume that $w^{1}$ and $w^{2}$ are G-matrices. Then $w^{1} \bigcirc w^{2}$ $\in M_{2}\left(B\left(H^{1} \otimes H^{2}\right)\right)$ is a G-matrix.

In what follows we assume that $|\mu|<1$. We end this section with the following version of Iwasawa decomposition.

Theorem 1.3. Let $w$ be an $S_{\mu} L(2, \mathbb{C})$-matrix. Then there exists unique decomposition of the form

$$
w=w_{c} w_{d},
$$

where $w_{c}$ is an $S_{\mu} U(2)$-matrix and $w_{d}$ is an $A N_{\mu}$-matrix. Matrix elements of $w_{c}$ doubly commute with matrix elements of $w_{d}$. Moreover the matrix elements of $w_{c}$ and $w_{d}$ belong to the $C^{*}$-algebra generated by matrix elements of $w$.

Proof. At first we notice that any $S_{\mu} L(2, \mathbb{C})$-matrix is invertible: Using (1.9)-(1.15) one can easily check that

$$
w^{-1}=\left(\begin{array}{cc}
\delta, & -\mu^{-1} \beta \\
-\mu \gamma, & \alpha
\end{array}\right)
$$

is the inverse of (1.27).

Let $p, q, r$ be elements of $B(H)$ such that

It means that

$$
w^{*} w=\left(\begin{array}{cc}
p, & q \\
q^{*}, & r
\end{array}\right) .
$$

$$
\begin{aligned}
& p=\alpha^{*} \alpha+\gamma^{*} \gamma, \\
& q=\alpha^{*} \beta+\gamma^{*} \delta, \\
& r=\beta^{*} \beta+\delta^{*} \delta,
\end{aligned}
$$

where $\alpha, \beta, \gamma$, and $\delta$ are matrix elements of $w$ (cf. (1.27)). Clearly $p$ is positive selfadjoint. Using relations (1.9)-(1.25) one can verify that

$$
\begin{gathered}
\alpha q=\mu q \alpha, \quad \alpha^{*} q=\mu q \alpha^{*}, \\
\gamma q=\mu q \gamma, \quad \gamma^{*} q=\mu q \gamma^{*}, \\
p \alpha=\alpha p, \quad p \gamma=\gamma p, \\
\delta p=\mu^{-2} \gamma q+\alpha^{*}, \\
\beta p=\mu^{-2} \alpha q-\mu \gamma^{*} . \\
r p=I+\mu^{-2} q^{*} q, \\
q p=\mu^{-2} p q .
\end{gathered}
$$

We claim that

Indeed using (1.44), (1.45), and (1.15) we have

$$
\begin{aligned}
r p & =\beta^{*} \beta p+\delta^{*} \delta p=\beta^{*}\left(\mu^{-2} \alpha q-\mu \gamma^{*}\right)+\delta^{*}\left(\mu^{-2} \gamma q+\alpha^{*}\right) \\
& =(\alpha \delta-\mu \beta \gamma)^{*}+\mu^{-2}\left(\beta^{*} \alpha+\delta^{*} \gamma\right) q=I+\mu^{-2} q^{*} q .
\end{aligned}
$$

Similarly one can prove (1.47). 
We shall also use the equality

$$
\mu^{-2} q^{*} q-q q^{*}=\left(1-\mu^{2}\right)\left(p^{2}-I\right) .
$$

The proof of this formula consists in complicated and boring computations. The difference

$$
\mu^{-2} q^{*} q-q q^{*}-\left(1-\mu^{2}\right)\left(p^{2}-I\right)
$$

is a linear combination of monomials of the form $a_{1}^{*} a_{2} a_{3}^{*} a_{4}$ (where $a_{k} \in\{\alpha, \beta, \gamma, \delta\}$, $k=1,2,3,4)$. Using (1.16)-(1.25) one may convert (1.49) into a linear combination of monomials of the form $a_{1}^{*} a_{2}^{*} a_{3} a_{4}$. Then using (1.9)-(1.15) one easily verifies that (1.49) vanishes. The details are left to the reader.

Let $S Q R_{\mu}: \mathbb{R}_{+} \rightarrow \mathbb{C}$ be the function such that $\left|S Q R_{\mu}(t)\right|=t^{1 / 2}$ and $S Q R_{\mu}(t) \in S_{\mu}$ for any $t \in \mathbb{R}_{+}$. Clearly $S Q R_{\mu}$ is continuous and

$$
S Q R_{\mu}\left(\mu^{-2} t\right)=\mu^{-1} S Q R_{\mu}(t)
$$

for all $t \in \mathbb{R}_{+}$. If $\mu>0$ then $S Q R_{\mu}$ coincides with the arithmetic square root.

Relation (1.46) shows that $p$ is invertible. Let

$$
a=S Q R_{\mu}(p) .
$$

Then $a$ is normal, $S p a \subset S_{\mu}$ and

$$
a^{*} a=p .
$$

Using (1.43), (1.47) and taking into account (1.50) we obtain

$$
\begin{gathered}
a \alpha=\alpha a, \quad a \gamma=\gamma a, \\
a^{*} \alpha=\alpha a^{*}, \quad a^{*} \gamma=\gamma a^{*}, \\
q a=\mu^{-1} a q, \quad q a^{*}=\mu^{-1} a^{*} q .
\end{gathered}
$$

According to (1.46) $p$ is invertible, so is $a$. Let

$$
n=a^{*-1} q .
$$

Using (1.54) and remembering that $a$ is normal we see that (1.35)-(1.37) hold. Moreover, by virtue of (1.47) and (1.52), (1.38) is equivalent to (1.48). It means that $w_{d}$ introduced by (1.34) is an $A N_{\mu}$-matrix.

It turns out that

$$
w_{d}^{*} w_{d}=\left(\begin{array}{cc}
p, & q \\
q^{*}, & r
\end{array}\right)=w^{*} w .
$$

The only non-trivial part of this equation (the equality of matrix elements standing in the right-lower corner) follows easily from (1.46) (cf. (1.52) and (1.47)). Remembering that $S_{\mu} L(2, \mathbb{C})$-matrices are invertible and using $(1.56)$ we conclude that the matrix

$$
w_{c}=w w_{d}^{-1}
$$

is unitary. Let $\alpha_{c}, \beta_{c}, \gamma_{c}$, and $\delta_{c}$ be matrix elements of $w_{c}$ :

$$
w_{c}=\left(\begin{array}{ll}
\alpha_{c}, & \beta_{c} \\
\gamma_{c}, & \delta_{c}
\end{array}\right) .
$$


Using (1.40) to compute $w_{d}^{-1}$ we get

$$
\begin{aligned}
& \alpha_{c}=\alpha a^{-1}, \\
& \beta_{c}=-\mu^{-1} \alpha n+\beta a, \\
& \gamma_{c}=\gamma a^{-1}, \\
& \delta_{c}=-\mu^{-1} \gamma n+\delta a .
\end{aligned}
$$

Multiplying both sides of (1.44) and (1.45) from the right by $a^{*-1}$ and using (1.53) and (1.54) we see that

$$
\begin{gathered}
\beta_{c}=-\mu \gamma_{c}^{*}, \\
\delta_{c}=\alpha_{c}^{*},
\end{gathered}
$$

so that $w_{c}$ is of the form (1.28). According to the remark following formula (1.33), $w_{c}$ is an $S_{\mu} U(2)$-matrix. Moreover using definitions (1.57), (1.58), (1.55) and taking into account (1.53), (1.54), (1.41), and (1.42) one can easily verify that $a$ and $n$ commute with $\alpha_{c}, \gamma_{c}, \alpha_{c}^{*}$, and $\gamma_{c}^{*}$.

To prove the uniqueness of the decomposition (1.39) we notice that due to the unitarity of $w_{c}$ we have $a^{*} a=p$ and $a^{*} n=q$. Using the first formula, remembering that $a$ is normal (cf.(1.35)) and taking into account the spectral condition we obtain (1.51). The second formula implies (1.55). It shows that $w_{d}$ is uniquely determined, so is $w_{c}$. Q.E.D.

\section{Compact Quantum Groups}

In the first part of this section we recall the basic results of the theory of compact quantum groups [10] mainly in order to introduce convenient notation. In particular we introduce the convolution product of "continuous functions" on a compact quantum group. In the second part we describe a discrete quantum space of all characters of any compact quantum group, implementing in this way the program announced in [8].

Let $G_{c}=\left(A_{c}, u^{c}\right)$ be a compact quantum group (i.e. compact matrix pseudogroup in the sense of [10]), $\mathscr{A}_{c}$ be the dense *-subalgebra of $A_{c}$ consisting of all smooth elements, $\Phi_{c}$ be the comultiplication, $\kappa_{c}$ be the coinverse and $e_{c}$ be the counit related to $G_{c}$. The subscript " $c$ " stands for "compact" and will distinguish $G_{c}$ among other quantum groups considered in this paper.

In [10] the convolution products of the form $a * \xi, \zeta * a$, and $\zeta * \xi$ (where $a \in \mathscr{A}_{c}$, $\zeta, \xi \in \mathscr{A}_{c}^{\prime}$ ) were considered. In the present paper we also need the convolution product of elements of $\mathscr{A}_{c}$. Let $h_{c}$ be the normalized Haar measure on $G_{c}$. For any $a \in \mathscr{A}_{c}$ we consider linear functionals $\varrho_{a}, \varrho_{a}^{\prime} \in A_{c}^{\prime}$ introduced by the formulae:

$$
\begin{aligned}
& \varrho_{a}(b)=h_{c}\left(b \kappa_{c}^{-1}(a)\right), \\
& \varrho_{a}^{\prime}(b)=h_{c}\left(b \kappa_{c}(a)\right),
\end{aligned}
$$

for any $b \in A_{c}$. Then the convolution product of two elements $a, b \in \mathscr{A}_{c}$ is defined by the formula

$$
a * b=a * \varrho_{b}=\varrho_{a}^{\prime} * b
$$


In order to check the second equality it is sufficient to assume that $a$ and $b$ are matrix elements of irreducible representations and use the orthogonality relations (cf. [10], Theorem 5.7). The simple computations are left to the reader.

Let us notice that the convolution product is associative:

$$
\begin{aligned}
& (b * a) * \xi=b *(a * \xi), \\
& \xi *(b * a)=(\xi * b) * a, \\
& (a * b) * c=a *(b * c),
\end{aligned}
$$

for any $a, b, c \in \mathscr{A}_{c}$ and $\xi \in \mathscr{A}_{c}^{\prime}$. Indeed we have $(b * a) * \xi=\left(\varrho_{b}^{\prime} * a\right) * \xi=\varrho_{b}^{\prime} *(a * \xi)$ $=b *(a * \xi)$ and similarly $\xi *(b * a)=\xi *\left(b * \varrho_{a}\right)=(\xi * b) * \varrho_{a}=(\xi * b) * a$. To prove (2.4) we notice that according to $(2.3) b *\left(\varrho_{a} * \xi\right)=b * \varrho_{a * \xi}$ for any $b \in \mathscr{A}_{c}$. Therefore

$$
\varrho_{a} * \xi=\varrho_{a * \xi}
$$

for any $a \in \mathscr{A}_{c}$ and $\xi \in \mathscr{A}_{c}^{\prime}$. Inserting $\xi=\varrho_{b}$ and using once more (2.2) we obtain

$$
\varrho_{a} * \varrho_{b}=\varrho_{a * b}
$$

for any $a, b \in \mathscr{A}_{c}$. Remembering that the convolution product of elements of $\mathscr{A}_{c}^{\prime}$ is associative we get

$$
\varrho_{(a * b) * c}=\varrho_{a *(b * c)}
$$

and (2.4) follows (the Haar measure restricted to $\mathscr{A}_{c}$ is faithful).

Let

$$
\left\{u^{\alpha}: \alpha \in \widehat{G}_{c}\right\}
$$

be the complete set of mutually non-equivalent irreducible unitary representations of $G_{c}$. The carrier Hilbert space of $u^{\alpha}$ will be denoted by $H^{\alpha}: u^{\alpha} \in B\left(H^{\alpha}\right) \otimes A_{c}$. We know that $\operatorname{dim} H^{\alpha}<\infty$. According to the general theory [10],

$$
\begin{gathered}
\left(\mathrm{id} \otimes \Phi_{c}\right) u^{\alpha}=u^{\alpha}\left(u^{\alpha},\right. \\
\left(\mathrm{id} \otimes \kappa_{c}\right) u^{\alpha}=\left(u^{\alpha}\right)^{*}, \\
\left(\mathrm{id} \otimes e_{c}\right) u^{\alpha}=I_{B\left(K^{\alpha}\right)} .
\end{gathered}
$$

Using the leg numbering notation one may write $u_{12}^{\alpha} u_{13}^{\alpha}$ instead of $u^{\alpha} \oplus u^{\alpha}$.

We recall ([10], Theorems 5.6 and 5.7) that in general the Haar measure on $G_{c}$ is not central. Its modular properties (in the sense of Tomita theory) are described by a holomorphic family $\left(f_{z}\right)_{z \in \mathbb{C}}$ of linear multiplicative functionals on $\mathscr{A}_{c}$. In particular the orthogonality relations for the matrix elements of $u^{\alpha}$ contain the operator $F_{\alpha} \in B\left(H^{\alpha}\right)$ introduced by the formula

$$
F_{\alpha}=\left(\mathrm{id} \otimes f_{1}\right) u^{\alpha} .
$$

It is known that $F_{\alpha}$ is positive invertible for any $\alpha \in \widehat{G}_{c^{*}}$.

Let

$$
A_{d}=\sum_{\alpha \in \hat{\hat{G}}_{c}} B\left(H^{\alpha}\right) .
$$

The canonical projections $A_{d} \rightarrow B\left(H^{\alpha}\right)$ will be denoted by $\pi_{\alpha}$. In general the sum (2.11) is infinite and $A_{d}$ has no unity. In what follows, $I_{d}$ will denote unity of $M\left(A_{d}\right)$. 
Let $A_{d 0}$ be the Pedersen ideal of $A_{d}: x \in A_{d 0}$ if and only if the set

$$
\left\{\alpha \in \widehat{G}_{c}: \pi_{\alpha}(x) \neq 0\right\}
$$

is finite. The above set will be called central support of $x$.

For any $x \in A_{d 0}$ we set

$$
\begin{aligned}
& h_{d L}(x)=\sum_{\alpha \in \hat{G}_{c}} M_{\alpha} \operatorname{Tr}_{\alpha}\left(F_{\alpha}^{-1} \pi_{\alpha}(x)\right), \\
& h_{d R}(x)=\sum_{\alpha \in \hat{\boldsymbol{G}}_{c}} M_{\alpha} \operatorname{Tr}_{\alpha}\left(F_{\alpha} \pi_{\alpha}(x)\right),
\end{aligned}
$$

where $F_{\alpha}$ is given by $(2.10), M_{\alpha}=\operatorname{Tr}_{\alpha} F_{\alpha}$ and $\operatorname{Tr}_{\alpha}$ denotes the trace on $B\left(H^{\alpha}\right)$ (normalized in such a way that $\operatorname{Tr}_{\alpha} I_{\alpha}=\operatorname{dim} H^{\alpha}$ ). Clearly $h_{d L}$ and $h_{d R}$ are positive linear functionals on $A_{d 0}$. We shall see later that $h_{d L}\left(h_{d R}\right.$ respectively) is left (right respectively) invariant Haar measure on the dual of $G_{c}$.

The following object plays the central role in our paper:

$$
u=\sum_{\alpha \in \hat{G}_{c}}^{\oplus} u^{\alpha} .
$$

Clearly $u$ is a unitary element of the multiplier algebra $M\left(A_{d} \otimes A_{c}\right)$. Relations (2.7)-(2.9) mean that

$$
\begin{gathered}
\left(\mathrm{id} \otimes \Phi_{c}\right) u=u_{12} u_{13}, \\
\left(\mathrm{id} \otimes \kappa_{c}\right) u=u^{*}, \\
\left(\mathrm{id} \otimes e_{c}\right) u=I_{d} .
\end{gathered}
$$

Let us notice that $u \in A_{d} \otimes_{r} \mathscr{A}_{c}$ (see Sect. 0 for the meaning of symbol $\otimes_{r}$ ) so the lefthand sides of (2.16) and (2.17) are well defined.

Now we shall briefly discuss the Fourier analysis on $G_{c}$. For each $a \in A_{c}$ we denote by $h_{c} a\left(a h_{c}\right.$ respectively) the linear functional on $A_{c}$ such that $\left(h_{c} a\right)(b)=h_{c}(a b)$ $\left(\left(a h_{c}\right)(b)=h_{c}(b a)\right.$ respectively) for any $b \in A_{c}$. Similarly for any $x \in A_{d 0}$ we introduce $h_{d L} x, h_{d R} x, x h_{d L}, x h_{d R} \in A_{d \cdot}^{\prime}$. The Fourier transform of an element $a \in \mathscr{A}_{c}$ is introduced by the formula

$$
\mathscr{F} a=\left(\mathrm{id} \otimes h_{c} a\right) u^{*} .
$$

Using the orthogonality relations for the matrix elements of irreducible representations of $G_{c}$ one can easily check that

$$
\mathscr{F}: \mathscr{A}_{c} \rightarrow A_{d 0}
$$

is a linear bijective mapping and that the inverse mapping

$$
\mathscr{F}^{-1}: A_{d 0} \rightarrow \mathscr{A}_{c}
$$

is given by the formula

$$
\mathscr{F}^{-1} x=\left(h_{d L} x \otimes \mathrm{id}\right) u .
$$

We have the usual formulae:

$$
\begin{aligned}
\mathscr{F}(a * b) & =\mathscr{F}(a) \mathscr{F}(b), \\
(\mathscr{F} a)^{*} & =\mathscr{F}\left(\kappa_{c}\left(a^{*}\right)\right),
\end{aligned}
$$


for any $a, b \in \mathscr{A}_{c}$. Relation (2.21) can be checked by direct computation: Using (2.18) and (2.16) we have $(\mathscr{F} a)^{*}=\left(\mathrm{id} \otimes a^{*} h_{c}\right) u=\left(\mathrm{id} \otimes\left(a^{*} h_{c}\right) \circ \kappa_{c}^{-1}\right) u^{*}$. On the other hand $\left(\left(a^{*} h_{c}\right) \circ \kappa_{c}^{-1}\right)(b)=\left(a^{*} h_{c}\right)\left(\kappa_{c}^{-1}(b)\right)=h_{c}\left(\kappa_{c}^{-1}(b) a^{*}\right)=h_{c}\left(\kappa_{c}\left(a^{*}\right) b\right)$ for any $b \in \mathscr{A}_{c}$ and (2.21) follows. To prove (2.20) we combine formulae (2.18) and (2.16). Taking into account (2.1) we obtain

$$
\mathscr{F} a=\left(\mathrm{id} \otimes \varrho_{a}\right) u .
$$

Now (2.20) follows from (2.15) and (2.6). Moreover using (2.5) instead of (2.6) we get

$$
\mathscr{F}(a * \xi)=(\mathscr{F} a)(\mathrm{id} \otimes \xi) u
$$

for any $a \in \mathscr{A}_{c}$ and $\xi \in \mathscr{A}_{c}^{\prime}$. Let us also notice that

$$
h_{d L}(\mathscr{F} a)=e_{c}(a)
$$

for any $a \in \mathscr{A}_{c}$. This formula follows immediately from (2.19) and (2.17).

Let $G_{d}$ be the quantum space (cf. [8]) related to the $C^{*}$-algebra $A_{d}$. Formula (2.15) means that $\left(G_{d}, u\right)$ is a quantum family of characters of $G_{c}$. The following theorem shows that $\left(G_{d}, u\right)$ is the quantum space of all characters of $G_{c}$ :

Theorem 2.1. Let $B$ be a $C^{*}$-algebra and $v$ be a unitary element of $M\left(B \otimes A_{c}\right)$ such that

$$
\left(\mathrm{id} \otimes \Phi_{c}\right) v=v_{12} v_{13} .
$$

Then there exists unique $\psi \in \operatorname{Mor}\left(A_{d}, B\right)$ such that

$$
v=(\psi \otimes \mathrm{id}) u .
$$

Proof. If (2.26) holds then for any $a \in \mathscr{A}_{c}$,

$$
\left(\mathrm{id} \otimes h_{c} a\right) v^{*}=\psi(\mathscr{F} a),
$$

and for any $x \in A_{d 0}$,

$$
\psi(x)=\mathscr{F}_{v} \circ \mathscr{F}^{-1}(x),
$$

where

$$
\mathscr{F}_{v}: \mathscr{A}_{c} \rightarrow M(B)
$$

is the linear mapping introduced by the formula: for any $a \in \mathscr{A}_{c}$,

$$
\mathscr{F}_{v} a=\left(\mathrm{id} \otimes h_{c} a\right) v^{*} .
$$

The uniqueness of $\psi$ is proved ( $A_{d 0}$ is dense in $A_{d}$ ). To prove the existence we consider the linear mapping

$$
\psi: A_{d 0} \rightarrow M(B)
$$

introduced by the formula (2.28). We shall prove that this mapping is a *-algebra homomorphism:

$$
\begin{gathered}
\psi(x y)=\psi(x) \psi(y), \\
\psi\left(z^{*}\right)=\psi(z)^{*},
\end{gathered}
$$

for any $x, y, z \in A_{d 0}$. 
We know $\left(h_{c}\right.$ is the Haar measure) that $\left(\mathrm{id} \otimes h_{c}\right) \Phi_{c}(x)=h_{c}(x) I_{c}$ for any $x \in A_{c}$. Therefore for any $a \in A_{c}$,

$$
\left(\mathrm{id} \otimes \mathrm{id} \otimes h_{c}\right)\left(\mathrm{id} \otimes \Phi_{c}\right)\left[(I \otimes a) v^{*}\right]=\left(\mathrm{id} \otimes h_{c}\right)\left[(I \otimes a) v^{*}\right] \otimes I_{c} .
$$

The right-hand side equals to $\mathscr{F}_{v}(a) \otimes I_{c}$. To compute the left-hand side we use multiplicativity of $\Phi$ and formula (2.25). We get

$$
\left(\mathrm{id} \otimes \mathrm{id} \otimes h_{c}\right)\left[\left(I \otimes \Phi_{c}(a)\right) v_{13}^{*}\right] v^{*}=\mathscr{F}_{v}(a) \otimes I_{c}
$$

and ( $v$ is unitary)

$$
\left(\mathrm{id} \otimes \mathrm{id} \otimes h_{c}\right)\left[\left(I \otimes \Phi_{c}(a)\right) v_{13}^{*}\right]=\left(\mathscr{F}_{v}(a) \otimes I_{c}\right) v .
$$

Let $b \in A_{c^{*}}$. Applying to both sides of the above equation (id $\otimes b^{*} h_{c}$ ) we obtain

$$
\mathscr{F}_{v}\left(a * b * h_{c}\right)=\mathscr{F}_{v}(a) \mathscr{F}_{v}(b)^{*} .
$$

Repeating the above computations with $v$ replaced by $u$ one obtains

$$
\mathscr{F}\left(a * b * h_{c}\right)=\mathscr{F}(a) \mathscr{F}(b)^{*} .
$$

Comparing the last two relations and taking into account $(2.28)$ we see that

$$
\psi\left(x y^{*}\right)=\psi(x) \psi(y)^{*}
$$

for any $x, y \in A_{d 0}$. Any element $z \in A_{d 0}$ is of the form $z=x y^{*}$, where $x, y \in A_{d 0}$. Then $z^{*}=y x^{*}$ and (2.32) follows immediately from (2.33). Moreover inserting in (2.33) $y^{*}$ instead of $y$ and using (2.32) (with $z$ replaced by $y$ ) we obtain (2.31).

This way we proved that $(2.30)$ is a homomorphism of $*$-algebras. Remembering that $A_{d 0}$ is a union of an increasing sequence of $C^{*}$-algebras we conclude that (2.30) is continuous and can be extended to $A_{d}$.

Let $a \in \mathscr{A}_{c}$. Using definition (2.1) we see that

$$
\varrho_{a}^{*}(b) \stackrel{\mathrm{df}}{=} \overline{\varrho_{a}\left(b^{*}\right)}=\overline{h_{c}\left(b^{*} \kappa_{c}^{-1}(a)\right)}=h_{c}\left(\kappa_{c}^{-1}(a)^{*} b\right)=h_{c}\left(\kappa_{c}\left(a^{*}\right) b\right)
$$

for any $b \in A_{c}$. Therefore (cf. (2.29))

$$
\left(\left(\mathrm{id} \otimes \varrho_{a}\right) v\right)^{*}=\left(\mathrm{id} \otimes \varrho_{a}^{*}\right) v^{*}=\mathscr{F}_{v}\left(\kappa_{c}\left(a^{*}\right)\right) .
$$

On the other hand using (2.28), (2.32), (2.21) and once more (2.28) we obtain

$$
\left(\mathscr{F}_{v} a\right)^{*}=\psi(\mathscr{F} a)^{*}=\psi\left((\mathscr{F} a)^{*}\right)=\psi\left(\mathscr{F}\left(\kappa_{c}\left(a^{*}\right)\right)\right)=\mathscr{F}_{v}\left(\kappa_{c}\left(a^{*}\right)\right) .
$$

Comparing the last two equalities we get

$$
\mathscr{F}_{v} a=\left(\mathrm{id} \otimes \varrho_{a}\right) v
$$

for any $a \in \mathscr{A}_{c}$.

Let $x \in B$. Then $x \otimes I_{c} \in B \otimes A_{c}$ and $\left(x \otimes I_{c}\right) v \in B \otimes A_{c}\left(v\right.$ is a multiplier of $\left.B \otimes A_{c}\right)$. Therefore for any $\varepsilon>0$ there exist $b_{1}, b_{2}, \ldots, b_{N} \in B$ and $a_{1}, a_{2}, \ldots, a_{N} \in \mathscr{A}_{c}\left(\mathscr{A}_{c}\right.$ is dense in $A_{c}$ ) such that

$$
\left\|\left(x \otimes I_{c}\right) v-\sum_{n} b_{n} \otimes a_{n}\right\|<\varepsilon .
$$


Then

$$
\left\|\left(x \otimes I_{c}\right)-\left(\sum_{n} b_{n} \otimes a_{n}\right) v^{*}\right\|<\varepsilon
$$

and (id $\otimes h_{c}$ does not increase the norm)

$$
\left\|x-\sum_{n} b_{n} \mathscr{F}_{v}\left(a_{n}\right)\right\|<\varepsilon .
$$

It shows that $B \mathscr{F}_{v}\left(\mathscr{A}_{c}\right)$ is dense in $B$, so are $B \psi\left(A_{d}\right)\left(\right.$ clearly $\left.B \psi\left(A_{d}\right) \supset B \mathscr{F}_{v}\left(\mathscr{A}_{c}\right)\right)$ and $\psi\left(A_{d}\right) B$. It means that $\psi$ satisfies the nondegeneracy condition and $\psi \in \operatorname{Mor}\left(A_{d}, B\right)$.

To end this proof we have to check (2.26). We know that (2.28) and consequently (2.27) hold. Let

Then for any $a \in \mathscr{A}_{c}$,

$$
v^{\prime}=(\psi \otimes \mathrm{id}) u
$$

$$
\mathscr{F}_{v} a=\mathscr{F}_{{ }^{\prime}} a .
$$

If the Haar measure $h_{c}$ were faithful then we could conclude that $v=v^{\prime}$. In the general case using (2.34) we have

$$
\left(\mathrm{id} \otimes \varrho_{a}\right) v=\left(\mathrm{id} \otimes \varrho_{a}\right) v^{\prime} .
$$

Inserting $a * \xi$ (where $\xi \in A_{c}^{\prime}$ ) instead of $a$ and taking into account (2.5) we obtain

$$
\left(\mathrm{id} \otimes \varrho_{a}\right) v(\mathrm{id} \otimes \xi) v=\left(\mathrm{id} \otimes \varrho_{a}\right) v^{\prime}(\mathrm{id} \otimes \xi) v^{\prime}=\left(\mathrm{id} \otimes \varrho_{a}\right) v(\mathrm{id} \otimes \xi) v^{\prime} .
$$

Remembering that $\left(\mathrm{id} \otimes \varrho_{a}\right) v=\mathscr{F}_{v}(a)$ and that $B \mathscr{F}_{v}\left(\mathscr{A}_{c}\right)$ is dense in $B$ we have

$$
(\mathrm{id} \otimes \xi) v=(\mathrm{id} \otimes \xi) v^{\prime}
$$

for any $\xi \in A^{\prime}$ and relation $v=v^{\prime}$ follows. Q.E.D.

Remark. If $B=C B(H)$ (the algebra of all compact operators acting on a Hilbert space $H$ ) then $v$ is (called) "strongly continuous" representation of $G_{c}$ acting on $H$. In this case it follows immediately from Theorem 2.1 that $v$ is an orthogonal direct sum of finite-dimensional irreducible representations. If moreover $v \in B(H) \otimes A_{c}$ then $v$ is (said to be) norm-continuous. In this case $\psi \in \operatorname{Mor}\left(A_{d}, B(H)\right)$, the nondegeneracy of $\psi$ means that there exists a central projection $E \in A_{d}$ such that $\psi(E)=I_{B(H)}$ and only a finite number of nonequivalent irreducible representations may enter into the decomposition of $v$.

\section{Duals of Compact Quantum Groups}

In this section we use the universal property (Theorem 2.1) to introduce the group structure on the quantum space $G_{d}$. We shall proceed according to the program described in [8]. All notions related to the group structure on $G_{d}$ will be given in an explicit way. It turns out that in the general case $G_{d}$ is not unimodular: The left Haar measure is neither right invariant nor central.

At first we shall prove that the quantum space $G_{d}$ carries a natural group structure. In the theorem formulated below, $s_{d d}$ denotes the flip automorphism of 
$A_{d} \otimes A_{d}$ and $m_{d}$ denotes the multiplication map defined on $A_{d} \otimes_{\mathrm{r}} A_{d}$ :

$$
\begin{gathered}
s_{d d}(x \otimes y)=y \otimes x, \\
m_{d}(x \otimes y)=x y,
\end{gathered}
$$

for any $x, y \in A_{d}$. Moreover we shall consider a proper linear mapping $\kappa_{d}$ acting on $A_{d 0}$. We recall that for any such mapping $\kappa_{d} \otimes \mathrm{id}_{\mathscr{A}_{c}}\left(\kappa_{d} \otimes \kappa_{d}, \kappa_{d} \otimes \mathrm{id}_{A_{d}}, \mathrm{id}_{A_{d}} \otimes \kappa_{d}\right.$ respectively) admits the natural extension to a linear mapping acting on $A_{d} \otimes_{r} \mathscr{A}_{c}$ $\left(A_{d} \otimes_{r} A_{d}\right.$ respectively). We shall also use the leg numbering notation.

\section{Theorem 3.1.}

1. There exists unique $\Phi_{d} \in \operatorname{Mor}\left(A_{d}, A_{d} \otimes A_{d}\right)$ such that

$$
\left(\Phi_{d} \otimes \mathrm{id}\right) u=u_{23} u_{13} .
$$

This morphism is coassociative, i.e.

$$
\left(\Phi_{d} \otimes \mathrm{id}\right) \Phi_{d}=\left(\mathrm{id} \otimes \Phi_{d}\right) \Phi_{d} .
$$

2. There exists unique $e_{d} \in \operatorname{Mor}\left(A_{d}, \mathbb{C}\right)$ (i.e. $e_{d}$ is a $*$-character) such that

$$
\left(e_{d} \otimes \mathrm{id}\right) u=I_{c} .
$$

$e_{d}$ and $\Phi_{d}$ are related by the equality

$$
\left(e_{d} \otimes \mathrm{id}\right) \Phi_{d}=\left(\mathrm{id} \otimes e_{d}\right) \Phi_{d}=\mathrm{id} .
$$

3. There exists unique linear antimultiplicative bijective proper mapping

such that

$$
\kappa_{d}: A_{d 0} \rightarrow A_{d 0}
$$

$$
\left(\kappa_{d} \otimes \mathrm{id}\right) u^{*}=u \text {. }
$$

Moreover for any $x \in A_{d}$ we have

$$
\begin{gathered}
\kappa_{d}\left(\kappa_{d}\left(x^{*}\right)^{*}\right)=x, \\
\Phi_{d}\left(\kappa_{d}(x)\right)=s_{d d}\left(\kappa_{d} \otimes \kappa_{d}\right) \Phi_{d}(x), \\
m_{d}\left(\kappa_{d} \otimes \mathrm{id}\right) \Phi_{d}(x)=e_{d}(x) I_{d}, \\
m_{d}\left(\mathrm{id} \otimes \kappa_{d}\right) \Phi_{d}(x)=e_{d}(x) I_{d} .
\end{gathered}
$$

In what follows, $\Phi_{d}\left(e_{d}, \kappa_{d}\right.$ respectively) will be called the comultiplication (counit, coinverse respectively) related to $G_{d}$.

Proof. To prove the existence and uniqueness of $\Phi_{d}$ we set in Theorem 2.1, $B=A_{d} \otimes A_{d}$ and $v=u_{23} u_{13}$. It is sufficient to verify condition (2.25).

Using multiplicativity of $\Phi_{c}$ and (2.15) we have

$$
\left(\mathrm{id} \otimes \Phi_{c}\right) v=u_{23} u_{24} u_{13} u_{14}=u_{23} u_{13} u_{24} u_{14}=v_{12} v_{13} .
$$

Setting $\Phi_{d}=\psi$ we see that (3.3) coincides with (2.26). In the same way one can prove the existence and uniqueness of $e_{d}$. In this case we take $B=\mathbb{C}$ and $v=I_{c}$. The reader can easily notice that $e_{d}$ coincides with $\pi_{0}$, where $0 \in \widehat{G}_{c}$ is the trivial representation of $G_{c^{*}}$ 
To show formulae (3.4) and (3.6) we notice that according to (3.3) and (3.5),

$$
\begin{aligned}
& \left(\left[\left(\Phi_{d} \otimes \mathrm{id}\right) \Phi_{d}\right] \otimes \mathrm{id}\right) u=u_{34} u_{24} u_{14}, \\
& \left(\left[\left(\mathrm{id} \otimes \Phi_{d}\right) \Phi_{d}\right] \otimes \mathrm{id}\right) u=u_{34} u_{24} u_{14}, \\
& \left(\left[\left(e_{d} \otimes \mathrm{id}\right) \Phi_{d}\right] \otimes \mathrm{id}\right) u=u, \\
& \left(\left[\left(\mathrm{id} \otimes e_{d}\right) \Phi_{d}\right] \otimes \mathrm{id}\right) u=u,
\end{aligned}
$$

and use the uniqueness statement in Theorem 2.1.

The uniqueness of (3.7) can be easily established. Indeed using (3.8) and (2.18) we have

$$
\kappa_{d}(\mathscr{F} a)=\left(\mathrm{id} \otimes h_{c} a\right) u
$$

for any $a \in \mathscr{A}_{c}$. To prove the existence we have to go deeper into the representation theory of compact quantum groups ([10], [11]).

It is known that for any representation $\alpha \in \hat{G}_{c}$ there exists a complex conjugate representation $\bar{\alpha} \in \widehat{G}_{c}$. The relation between $\alpha$ and $\bar{\alpha}$ is described by an antilinear invertible mapping

$$
j_{\alpha}: H^{\alpha} \rightarrow H^{\bar{\alpha}} .
$$

This mapping induces the antilinear multiplicative mapping

$$
B\left(H^{\alpha}\right) \ni m \rightarrow m^{j} \in B\left(H^{\bar{\alpha}}\right)
$$

introduced by the formula $m^{j}=j_{\alpha} m j_{\alpha}^{-1}$. It is also known that $j_{\bar{\alpha}} j_{\alpha}=I_{B\left(H^{\alpha}\right)}$, and consequently for any $m \in B\left(H^{\alpha}\right)$,

$$
\left(m^{j}\right)^{j}=m .
$$

With this notation we have (cf. [11] p. 55, last formula)

$$
u^{\bar{\alpha}}=\left(u^{\alpha}\right)^{j \otimes *} \text {. }
$$

For any $x \in A_{d 0}$ we set

$$
\kappa_{d}(x)=\sum_{\alpha \in \hat{G}_{c}}^{\oplus}\left(\pi_{\alpha}\left(x^{*}\right)\right)^{j} .
$$

According to this definition $\kappa_{d}(x)$ is an element of $A_{d 0}$ such that

$$
\pi_{\bar{\alpha}}\left(\kappa_{d}(x)\right)=\left(\pi_{\alpha}\left(x^{*}\right)\right)^{j}
$$

for any $\alpha \in \widehat{G}_{c}$. Clearly $\kappa_{d}$ is linear (as a composition of two antilinear mappings) and antimultiplicative (hermitian conjugation is antimultiplicative and (3.13) is multiplicative). Using (3.14) one can easily verify (3.9). It shows that $\kappa_{d}$ is bijective.

We shall prove (3.8). Using (3.16) one can easily check that

$$
\left(\pi_{\bar{\alpha}} \circ \kappa_{d} \otimes \mathrm{id}\right) u^{*}=\sum m_{i}^{j} \otimes u_{i}^{*},
$$

where $m_{i}$ and $u_{i}$ are elements of $B\left(H^{\alpha}\right)$ and $\mathscr{A}_{c}$ respectively such that $\sum m_{i} \otimes u_{i}=u^{\alpha}$. Taking into account (3.15) we obtain

$$
\left(\pi_{\bar{\alpha}} \circ \kappa_{d} \otimes \mathrm{id}\right) u^{*}=u^{\bar{\alpha}}=\left(\pi_{\bar{\alpha}} \otimes \mathrm{id}\right) u
$$

for any $\alpha \in \widehat{G}_{c}$ and (3.8) follows. 
We shall prove (3.10). Using (3.8), (3.3), and (3.1) we have

$$
\begin{aligned}
& \left(\left[s_{d d}\left(\kappa_{d} \otimes \kappa_{d}\right) \Phi_{d} \circ \kappa_{d}^{-1}\right] \otimes \mathrm{id}\right) u=\left(\left[s_{d d}\left(\kappa_{d} \otimes \kappa_{d}\right) \Phi_{d}\right] \otimes \mathrm{id}\right) u^{*} \\
& \quad=\left(\left[s_{d d}\left(\kappa_{d} \otimes \kappa_{d}\right)\right] \otimes \mathrm{id}\right) u_{13}^{*} u_{23}^{*}=\left(s_{d d} \otimes \mathrm{id}\right) u_{13} u_{23} \\
& \quad=u_{23} u_{13}=\left(\Phi_{d} \otimes \mathrm{id}\right) u .
\end{aligned}
$$

Therefore $s_{d d}\left(\kappa_{d} \otimes \kappa_{d}\right) \Phi_{d} \circ \kappa_{d}^{-1}=\Phi_{d}$ and (3.10) follows. In a similar way one can prove (3.11) and (3.12). Using (3.3), (3.8), (3.2), unitarity of $u$ and (3.5) we have

$$
\begin{array}{r}
\left(\left[m_{d}\left(\kappa_{d} \otimes \mathrm{id}\right) \Phi_{d}\right] \otimes \mathrm{id}\right) u^{*}=\left(\left[m_{d}\left(\kappa_{d} \otimes \mathrm{id}\right)\right] \otimes \mathrm{id}\right) u_{13}^{*} u_{23}^{*} \\
=\left(m_{d} \otimes \mathrm{id}\right) u_{13} u_{23}^{*}=u u^{*}=I_{d} \otimes I_{c}=I_{d} \otimes\left(e_{d} \otimes \mathrm{id}\right) u^{*}, \\
\left(\left[m_{d}\left(\mathrm{id} \otimes \kappa_{d}\right) \Phi_{d}\right] \otimes \mathrm{id}\right) u^{*}=\left(\left[m_{d}\left(\mathrm{id} \otimes \kappa_{d}\right)\right] \otimes \mathrm{id}\right) u_{13}^{*} u_{23}^{*} \\
\quad=\left(m_{d} \otimes \mathrm{id}\right) u_{13}^{*} u_{23}=u^{*} u=I_{d} \otimes I_{c}=I_{d} \otimes\left(e_{d} \otimes \mathrm{id}\right) u^{*}
\end{array}
$$

and (3.11) and (3.12) follow. Q.E.D.

Applying $e_{d}$ to both sides of (2.18) and using (3.5) we obtain the dual version of (2.24):

$$
e_{d}(\mathscr{F} a)=h_{c}(a)
$$

for any $a \in \mathscr{A}_{c}$.

$G_{d}$ endowed with the comultiplication, counit and coinverse introduced in Theorem 3.1 is a non-compact discrete quantum group. It is the Pontryagin dual of $G_{c}$ [8]. The essence of the duality is contained in the unitary $u$. In order to stress the properties (2.15) and (3.3), we say that $u$ is a $\left(G_{d}, G_{c}\right)$-bicharacter. In a more standard approach (cf. [3]) the $\left(G_{d}, G_{c}\right)$ duality is described by a bilinear form $\langle$, defined on $A_{d} \times \mathscr{A}_{c}$. The relation of this form with the bicharacter $u$ is the following:

One can easily check that any element $x \eta A_{d}$ is of the form $x=(\operatorname{id} \otimes \xi) u$, where $\xi \in \mathscr{A}_{c}^{\prime}$ is uniquely determined. Then

$$
\langle x, a\rangle \stackrel{\text { df }}{=} \xi(a)
$$

for any $a \in \mathscr{A}_{c}$.

Now we shall show that $h_{d L}$ and $h_{d R}$ introduced by (2.12) and (2.13) are left and right respectively Haar measures on $G_{d}$.

For any $\alpha, \beta, \gamma \in \hat{G}_{c}$ we set

$$
\tau_{\alpha}^{\gamma \beta}=\left.\left(\pi_{\gamma} \otimes \pi_{\beta}\right) \Phi_{d}\right|_{B\left(H^{\alpha}\right)} .
$$

Clearly $\tau_{\alpha}^{\gamma \beta}$ is a (non-unital in general) *-algebra homomorphism

$$
\tau_{\alpha}^{\gamma \beta}: B\left(H^{\alpha}\right) \rightarrow B\left(H^{\gamma} \otimes H^{\beta}\right)
$$

It follows immediately from (2.14) and (3.3) that

$$
u^{\gamma *} \odot u^{\beta *}=\sum_{\alpha \in \hat{G}_{c}}^{\oplus}\left(\tau_{\alpha}^{\gamma \beta} \otimes \mathrm{id}\right) u^{\alpha *} .
$$


Proposition 3.2. For any $\alpha, \beta, \gamma$ the following conditions are equivalent;

(i) $\tau_{\alpha}^{\gamma \beta}$ is an embedding,

(ii) $\alpha \subset \beta \otimes \gamma$,

(iii) $\gamma \subset \bar{\beta} \otimes \alpha$,

(iv) $\beta C \alpha \otimes \bar{\gamma}$.

Proof. Taking into account the orthogonality relations for matrix elements of irreducible representations we see that $\alpha \subset \beta \otimes \gamma$ if and only if there exist matrix elements $a, b, c$ of $u^{\alpha}, u^{\beta}$, and $u^{\gamma}$ respectively such that $h_{c}\left(a^{*} b c\right) \neq 0$. On the other hand by virtue of (3.19) $\tau_{\alpha}^{\gamma \beta} \neq 0$ if and only if $h_{c}\left(c^{*} b^{*} a\right) \neq 0$ for some $a, b, c$ as above. We also know that $\tau_{\alpha}^{\gamma \beta} \neq 0$ if and only if $\tau_{\alpha}^{\gamma \beta}$ is an embedding $\left(B\left(H^{\alpha}\right)\right.$ is simple). The equivalence (i) $\Leftrightarrow$ (ii) is proved.

We shall use notation of [11]. If $\alpha \subset \beta \otimes \gamma$ then there exists non-zero $s \in \operatorname{Mor}(\alpha, \beta \otimes \gamma)$. In this case $I_{\bar{\beta}} \otimes s \in \operatorname{Mor}(\bar{\beta} \otimes \alpha, \quad \bar{\beta} \otimes \beta \otimes \gamma) \quad$ and $\left(\bar{t} \otimes I_{\gamma}\right)\left(I_{\bar{\beta}} \otimes s\right) \in \operatorname{Mor}(\bar{\beta} \otimes \alpha, \gamma)$, where $\bar{t} \in \operatorname{Mor}(\bar{\beta} \otimes \beta$, trivial representation) is given by the formula

$$
\bar{t}(k \otimes l)=\left(j_{\beta}^{-1}(k) \mid l\right)_{H^{\beta}}
$$

for any $k \in H^{\bar{\beta}}$ and $l \in H^{\beta}$. Using this formula one can easily check that $\left(\bar{t} \otimes I_{\gamma}\right)\left(I_{\bar{\beta}} \otimes s\right) \neq 0$. Remembering that $\gamma$ is irreducible we see that $\gamma \subset \bar{\beta} \otimes \alpha$ and the implication (ii) $\Rightarrow$ (iii) is proved. In the same way one checks implications (iii) $\Rightarrow$ (ii) and (ii) $\Leftrightarrow$ (iv). Q.E.D.

It follows immediately from Proposition 3.2 that for any $\alpha, \beta \in \hat{G}_{c}$ we have $\tau_{\alpha}^{\beta \gamma}=0$ and $\tau_{\alpha}^{\gamma \beta}=0$ for almost all $\gamma \in \widehat{G}_{c}$ ("almost all" means "all except a finite number of"). Taking into account (3.18) we see that for any $x \in A_{d 0}$ and any $\beta \in \hat{G}_{c}$,

$$
\begin{gathered}
\left(\pi_{\beta} \otimes \pi_{y}\right) \Phi_{d}(x)=0, \\
\left(\pi_{\gamma} \otimes \pi_{\beta}\right) \Phi_{d}(x)=0
\end{gathered}
$$

for almost all $\gamma \in \widehat{G}_{c}$. Due to this property we may apply id $\otimes h_{d L}, \mathrm{id} \otimes h_{d R}, h_{d L} \otimes \mathrm{id}$, $h_{d R} \otimes$ id to $\Phi_{d}(x)$ (for $x \in A_{d 0}$ ).

Let $F$ be the element affiliated to $A_{d}$ such that $\pi_{\alpha}(F)=F_{\alpha}$ and let $\left(f_{z}\right)_{z \in \mathbb{C}}$ be the family of linear multiplicative functionals on $\mathscr{A}_{c}$ describing the modular properties of $h_{c}$. Using Theorem 5.6 of [10] one can verify that for any $t \in \mathbb{C}$,

$$
\begin{aligned}
F^{t} & =\left(\mathrm{id} \otimes f_{t}\right) u \\
& =\left(\mathrm{id} \otimes f_{-t}\right) u^{*} .
\end{aligned}
$$

We shall prove

Theorem 3.3. For any $x \in A_{d 0}$ we have

$$
\begin{gathered}
\left(\mathrm{id} \otimes h_{d L}\right) \Phi_{d}(x)=h_{d L}(x) I_{d}, \\
\left(h_{d L} \otimes \mathrm{id}\right) \Phi_{d}(x)=h_{d L}(x) F^{2}, \\
\left(\mathrm{id} \otimes h_{d R}\right) \Phi_{d}(x)=h_{d R}(x) F^{-2}, \\
\left(h_{d R} \otimes \mathrm{id}\right) \Phi_{d}(x)=h_{d R}(x) I_{d} .
\end{gathered}
$$


Proof. For any $x \in A_{d 0}$ and any $t \in \mathbb{R}$ we set

$$
h_{d}^{t}(x)=\sum_{\alpha \in \hat{G}_{c}} M_{\alpha} \operatorname{Tr}_{\alpha}\left(F_{\alpha}^{t} \pi_{\alpha}(x)\right)
$$

Then $h_{d L}=h_{d}^{-1}$ and $h_{d R}=h_{d}^{1}$. We claim that for any $a \in \mathscr{A}_{c}$,

$$
h_{d}^{t}(\mathscr{F} a)=f_{t+1}(a) .
$$

Indeed using (2.12), (3.20), (2.23), and (2.24) we have

$$
h_{d}^{t}(\mathscr{F} a)=h_{d L}\left((\mathscr{F} a) F^{t+1}\right)=h_{d L}\left(\mathscr{F}\left(a * f_{t+1}\right)\right)=e_{c}\left(a * f_{t+1}\right)=f_{t+1}(a) .
$$

Let $\zeta$ be a proper linear functional on $A_{d}(\zeta(x)$ depends only on finite number of components of $\left.x \in A_{d}\right)$ and $a \in \mathscr{A}_{c}$. Then

$$
\Phi_{d}(\mathscr{F} a)=\Phi_{d}\left(\mathrm{id} \otimes h_{c} a\right) u^{*}=\left(\mathrm{id} \otimes \mathrm{id} \otimes h_{c} a\right) u_{13}^{*} u_{23}^{*} .
$$

Applying to both sides $\zeta \otimes$ id we obtain

$$
(\zeta \otimes \mathrm{id}) \Phi_{d}(\mathscr{F} a)=\mathscr{F}\left(a(\zeta \otimes \mathrm{id}) u^{*}\right) .
$$

Using (3.26), remembering that $f_{z}$ are multiplicative and taking into account (3.21) we have

$$
\left(\zeta \otimes h_{d}^{t}\right) \Phi_{d}(\mathscr{F} a)=h_{d}^{t}(\mathscr{F} a) \zeta\left(F^{-t-1}\right) .
$$

This way we proved that

$$
\left(\mathrm{id} \otimes h_{d}^{t}\right) \Phi_{d}(x)=h_{d}^{t}(x) F^{-t-1}
$$

for any $x \in A_{d 0}$. Inserting $t= \pm 1$ we obtain (3.22) and (3.24). In order to prove (3.23) and (3.25) we proceed in a similar way. Applying (id $\otimes \zeta)$ to both sides of (3.27) we get

$$
(\mathrm{id} \otimes \zeta) \Phi_{d}(\mathscr{F} a)=\left(\mathrm{id} \otimes \mathrm{id} \otimes b h_{c} a\right) u^{*},
$$

where $b=(\zeta \otimes \mathrm{id}) u^{*}$. Trying to rewrite the right-hand side of this equation as a Fourier transform of some element of $\mathscr{A}_{c}$ we use the modular properties of $h_{c}: b h_{c}$ $=h_{c} b^{\prime}$, where $b^{\prime}=f_{-1} * b * f_{-1}=(F \zeta F \otimes \mathrm{id}) u^{*}$. Therefore we have

$$
(\mathrm{id} \otimes \zeta) \Phi_{d}(\mathscr{F} a)=\mathscr{F}\left(\left[(F \zeta F \otimes \mathrm{id}) u^{*}\right] a\right),
$$

and instead of (3.28) we obtain

$$
\left(h_{d}^{t} \otimes \mathrm{id}\right) \Phi_{d}(x)=h_{d}^{t}(x) F^{-t+1}
$$

for any $x \in A_{d 0}$. Inserting $t= \pm 1$ we obtain (3.23) and (3.25). Q.E.D.

Remark. Relation (3.22) ((3.25) respectively) shows that $h_{d L}$ is left ( $h_{d R}$ is right respectively) Haar measure on $G_{d}$. Let us notice that $h_{d L}$ is not invariant under right shifts: formula (3.23) shows that the corresponding modular function should be identified with $F^{2}$. We also have the following nice formula:

$$
h_{d L}\left(\kappa_{d}(x)\right)=h_{d R}(x)
$$

for any $x \in A_{d 0}$. The proof is left to the reader. 
Hint: Show that

$$
\kappa_{d}(\mathscr{F} a)=\mathscr{F}\left(\kappa_{c}\left(f_{1} * a * f_{1}\right)\right)
$$

for any $a \in \mathscr{A}_{c}$.

Applying $e_{d}$ to both sides of (3.29) and using (3.6) and (3.17) we get

$$
\zeta(\mathscr{F} a)=\left(F \zeta F \otimes h_{c}\right)\left[u^{*}\left(I_{d} \otimes a\right)\right] .
$$

Let $x \in A_{d 0}$. Inserting in the above relation $h_{d L} x=F^{-1} x F h_{d L}$ instead of $\zeta$ and using (2.18) and (2.13) we obtain

$$
\left(h_{d L} \otimes h_{c}\right)\left[(x \otimes a) u^{*}\right]=\left(h_{d R} \otimes h_{c}\right)\left[u^{*}(x \otimes a)\right] .
$$

Clearly $x \otimes a$ may be replaced by any element of the Pedersen ideal of $A_{d} \otimes A_{c}$. Inserting $u(x \otimes a)$ instead of $x \otimes a$ we get

$$
\left(h_{d L} \otimes h_{c}\right)\left[u(x \otimes a) u^{*}\right]=\left(h_{d R} \otimes h_{c}\right)(x \otimes a)
$$

for any $x \in A_{d 0}$ and $a \in \mathscr{A}_{c}$.

At the end of this section we shall show that there exists a natural $1-1$ correspondence between representations of $G_{d}$ acting on a finite-dimensional vector space $K$ and homomorphisms of $\mathscr{A}_{c}$ into $B(K)$.

Let $K$ be a finite-dimensional complex vector space and

$$
v_{d} \eta B(K) \otimes A_{d} .
$$

Then $v_{d}$ is of the form

$$
v_{d}=\sum_{i} m^{i} \otimes x_{i}
$$

where $m^{1}, m^{2}, \ldots, m^{(\operatorname{dim} K)^{2}}$ is a basis in $B(K)$ and $x_{i} \eta A_{d}\left(i=1,2, \ldots(\operatorname{dim} K)^{2}\right)$. We know that each $x_{i}$ is of the form

$$
x_{i}=\left(\mathrm{id} \otimes \xi_{i}\right) u^{*},
$$

where $\xi_{i} \in \mathscr{A}_{c}^{\prime}$. For any $a \in \mathscr{A}_{c}$ we set

$$
\xi(a)=\sum_{i} \xi_{i}(a) m^{i} .
$$

Then $\xi$ is a linear mapping from $\mathscr{A}_{c}$ into $B(K)$. Clearly any linear mapping from $\mathscr{A}_{c}$ into $B(K)$ is of this form and corresponding $v_{d} \eta B(K) \otimes A_{d}$ is uniquely determined.

Theorem 3.4. Let $K, v_{d}$, and $\xi$ be as above. Then the following two conditions are equivalent

1. $v_{d}$ is a nondegenerate representation of $G_{d}$ acting on $K$, i.e.

$$
\left(\mathrm{id} \otimes e_{d}\right) v_{d}=I_{B(K)}
$$

and

$$
\left(\mathrm{id} \otimes \Phi_{d}\right) v_{d}=v_{d 12} v_{d 13} .
$$

2. $\xi$ is an algebra homomorphism acting from $\mathscr{A}_{c}$ into $B(K)$, i.e.

$$
\xi\left(I_{c}\right)=I_{B(K)}
$$


and

$$
\xi(a b)=\xi(a) \xi(b)
$$

for any $a, b \in \mathscr{A}_{c}$.

Proof. It follows immediately from (3.32) and (3.5) that $e_{d}\left(x_{i}\right)=\xi_{i}\left(I_{c}\right)$. Therefore $\left(\mathrm{id} \otimes e_{d}\right) v_{d}=\sum m^{i} \xi_{i}\left(I_{c}\right)=\xi\left(I_{c}\right)$. It shows that formulae (3.34) and (3.36) are equivalent.

Let $c_{k}^{i j}\left(i, j, k=1,2, \ldots(\operatorname{dim} K)^{2}\right)$ be structure constants of $B(K)$ :

$$
m^{i} m^{j}=\sum_{k} c_{k}^{i j} m^{k}
$$

Then (3.35) is equivalent to the relation

$$
\Phi_{d}\left(x_{k}\right)=\sum_{i, j} c_{k}^{i j} x_{i} \otimes x_{j}
$$

whereas (3.37) is equivalent to the relation

$$
\xi_{k}(a b)=\sum_{i, j} c_{k}^{i j} \xi_{i}(a) \xi_{j}(b)
$$

Assume that (3.39) holds. Then using (3.32) and (3.3) we have

$$
\begin{aligned}
\Phi_{d}\left(x_{k}\right)=\left(\mathrm{id} \otimes \mathrm{id} \otimes \xi_{k}\right) u_{13}^{*} u_{23}^{*} & =\sum_{i, j} c_{k}^{i j}\left[\left(\mathrm{id} \otimes \xi_{i}\right)\left(u^{*}\right)\right] \otimes\left[\left(\mathrm{id} \otimes \xi_{j}\right)\left(u^{*}\right)\right] \\
& =\sum_{i, j} c_{k}^{i j} x_{i} \otimes x_{j}
\end{aligned}
$$

and (3.38) is proved. Conversely if (3.38) holds then repeating the above computations we show that

$$
\left(\mathrm{id} \otimes \mathrm{id} \otimes \xi_{k}\right) u_{13}^{*} u_{23}^{*}=\sum_{i, j} c_{k}^{i j}\left[\left(\mathrm{id} \otimes \xi_{i}\right) u^{*}\right] \otimes\left[\left(\mathrm{id} \otimes \xi_{j}\right) u^{*}\right]
$$

and (3.39) follows. Q.E.D.

Remark. One can easily check that the correspondence $v_{d} \leftrightarrow \xi$ is natural in the following sense: If $v_{d}^{1}$ and $v_{d}^{2}$ are non-degenerate representations of $D_{d}$ acting on finite-dimensional complex vector spaces $K_{1}$ and $K_{2}$ and $\xi^{1}, \xi^{2}$ are corresponding algebra homomorphisms then the set of intertwining operators

$$
\left\{t \in B\left(K_{1}, K_{2}\right):\left(t \otimes I_{d}\right) v_{d}^{1}=v_{d}^{2}\left(t \otimes I_{d}\right)\right\}
$$

coincides with

$$
\left\{t \in B\left(K_{1}, K_{2}\right): t \xi^{1}(a)=\xi^{2}(a) t \text { for all } a \in \mathscr{A}_{c}\right\}
$$

\section{Double Groups}

In this section we construct a quantum group $G$ that contains $G_{c}$ and $G_{d}$ as subgroups. As a quantum space, $G$ is homeomorphic to the cartesian product of $G_{c}$ and $G_{d}$, however as far as the group structure is considered, $G_{c}$ and $G_{d}$ are placed in $G$ in a nontrivial manner. 
In the next section we shall see that in the case $G_{c}=S_{\mu} U(2)$ the group $G$ should be identified with the quantum Lorentz group.

To understand better the content of this section we consider the classical case. Let $G$ be a locally compact topological group and $G_{c}, G_{d}$ be closed subgroups of $G$. Assume that the mappings

$$
\begin{aligned}
& \phi: G_{c} \times G_{d} \ni(x, y) \rightarrow x \cdot y \in G, \\
& \psi: G_{d} \times G_{c} \ni(y, x) \rightarrow y \cdot x \in G
\end{aligned}
$$

are homeomorphisms. Let $\sigma_{*}=\phi^{-1} \circ \psi$. Then

$$
\sigma_{*}: G_{d} \times G_{c} \rightarrow G_{c} \times G_{d}
$$

is a homeomorphism. It turns out that $\sigma_{*}$ cannot be arbitrary. It must be compatible with the multiplication rules in $G_{c}$ and $G_{d}$. In particular denoting by $e_{c *} \in G_{c}$ and $e_{d *} \in G_{d}$ the neutral elements we have

$$
\begin{gathered}
\sigma_{*}\left(e_{d *}, x\right)=\left(x, e_{d *}\right), \\
\sigma_{*}\left(y, e_{c *}\right)=\left(e_{c *}, y\right)
\end{gathered}
$$

for any $x \in G_{c}$ and $y \in G_{d}$. Moreover if $\Phi_{c *}$ and $\Phi_{d *}$ denote the multiplications in $G_{c}$ and $G_{d}$ respectively:

$$
\begin{aligned}
& \Phi_{c *}: G_{c} \times G_{c} \ni\left(x_{1}, x_{2}\right) \rightarrow x_{1} x_{2} \in G_{c}, \\
& \Phi_{d *}: G_{d} \times G_{d} \ni\left(y_{1}, y_{2}\right) \rightarrow y_{1} y_{2} \in G_{d},
\end{aligned}
$$

then one can easily check that

$$
\begin{aligned}
& \sigma_{*}\left(\mathrm{id} \times \Phi_{c *}\right)=\left(\Phi_{c *} \times \mathrm{id}\right)\left(\mathrm{id} \times \sigma_{*}\right)\left(\sigma_{*} \times \mathrm{id}\right), \\
& \sigma_{*}\left(\Phi_{d *} \times \mathrm{id}\right)=\left(\mathrm{id} \times \Phi_{d *}\right)\left(\sigma_{*} \times \mathrm{id}\right)\left(\mathrm{id} \times \sigma_{*}\right) .
\end{aligned}
$$

Conversely assume that locally compact topological groups $G_{c}$ and $G_{d}$ and a homeomorphism (4.1) satisfying conditions (4.2)-(4.5) are given. Then setting

$$
\left(x_{1}, y_{1}\right)\left(x_{2}, y_{2}\right)=\left(x_{1} x_{2}^{\prime}, y_{1}^{\prime} y_{2}\right) \text {, }
$$

where $x_{1}, x_{2} \in G_{c}, y_{1}, y_{2} \in G_{d}$ and $\left(x_{2}^{\prime}, y_{1}^{\prime}\right)=\sigma_{*}\left(y_{1}, x_{2}\right)$, we introduce a multiplication (binary operation) on $G=G_{c} \times G_{d}$ and $G$ endowed with this multiplication is a locally compact topological group. The neutral element $e_{*}$ of $G$ and the inverse of an element of $G$ are given by the formulae

$$
\begin{gathered}
e_{*}=\left(e_{* c}, e_{* d}\right), \\
(x, y)^{-1}=\sigma_{*}\left(y^{-1}, x^{-1}\right) .
\end{gathered}
$$

We say that the group $G$ is the twisted product of $G_{c}$ and $G_{d}$.

In this section we show that the twisted product construction can be done for quantum groups. We restrict ourselves to the case when $G_{c}$ is a compact quantum group and $G_{d}$ is the Pontriagin dual of $G_{c}$. In this case there exists a natural homeomorphism (4.1). We shall use the notation introduced in Sects. 2 and 3.

Let

$$
\sigma: A_{c} \otimes A_{d} \rightarrow A_{d} \otimes A_{c}
$$


be the *-algebra isomorphism introduced by the formula:

$$
\sigma(a \otimes x)=u(x \otimes a) u^{*}
$$

for any $a \in A_{c}$ and $x \in A_{d}$.

It follows immediately from (2.17) and (3.5) that

$$
\begin{aligned}
& \left(e_{d} \otimes \mathrm{id}\right) \sigma=\mathrm{id} \otimes e_{d}, \\
& \left(\mathrm{id} \otimes e_{c}\right) \sigma=e_{c} \otimes \mathrm{id} .
\end{aligned}
$$

These formulae correspond to (4.2) and (4.3). We shall prove (cf. (4.4) and (4.5)) that

$$
\begin{aligned}
& (\mathrm{id} \otimes \sigma)(\sigma \otimes \mathrm{id})\left(\mathrm{id} \otimes \Phi_{d}\right)=\left(\Phi_{d} \otimes \mathrm{id}\right) \sigma, \\
& (\sigma \otimes \mathrm{id})(\mathrm{id} \otimes \sigma)\left(\Phi_{c} \otimes \mathrm{id}\right)=\left(\mathrm{id} \otimes \Phi_{c}\right) \sigma .
\end{aligned}
$$

Assume that $a \in A_{c}$ and $x_{1}, x_{2} \in A_{d}$. Using definition (4.9) we obtain

$$
\begin{aligned}
(\mathrm{id} \otimes \sigma)(\sigma \otimes \mathrm{id})\left(a \otimes x_{1} \otimes x_{2}\right) & =(\mathrm{id} \otimes \sigma) u_{12}\left(x_{1} \otimes a \otimes x_{2}\right) u_{12}^{*} \\
& =u_{23} u_{13}\left(x_{1} \otimes x_{2} \otimes a\right) u_{13}^{*} u_{23}^{*} \\
& =\left[\left(\Phi_{d} \otimes \mathrm{id}\right) u\right]\left(x_{1} \otimes x_{2} \otimes a\right)\left[\left(\Phi_{d} \otimes \mathrm{id}\right) \mathrm{u}^{*}\right] .
\end{aligned}
$$

Therefore for any $a \in A_{c}$ and $x \in A_{d}$ we have $\left.(\mathrm{id} \otimes \sigma)(\sigma \otimes \mathrm{id})\left(a \otimes \Phi_{d}(x)\right)=\left[\left(\Phi_{d} \otimes \mathrm{id}\right) u\right]\left(\Phi_{d}(x) \otimes a\right)\left[\left(\Phi_{d} \otimes \mathrm{id}\right) u^{*}\right)\right]$ $=\left(\Phi_{d} \otimes \mathrm{id}\right)\left[u(x \otimes a) u^{*}\right]=\left(\Phi_{d} \otimes \mathrm{id}\right) \sigma(a \otimes x)$

and (4.12) follows. Similarly we prove (4.13). For any $a_{1}, a_{2} \in A_{c}$ and $x \in A_{d}$ we have $(\sigma \otimes \mathrm{id})(\mathrm{id} \otimes \sigma)\left(a_{1} \otimes a_{2} \otimes x\right)=(\sigma \otimes \mathrm{id}) u_{23}\left(a_{1} \otimes x \otimes a_{2}\right) u_{23}^{*}$

$$
\begin{aligned}
& =u_{12} u_{13}\left(x \otimes a_{1} \otimes a_{2}\right) u_{13}^{*} u_{12}^{*} \\
& =\left[\left(\mathrm{id} \otimes \Phi_{c}\right)(u)\right]\left(x \otimes a_{1} \otimes a_{2}\right)\left[\left(\mathrm{id} \otimes \Phi_{c}\right) u\right] .
\end{aligned}
$$

Therefore for any $a \in A_{c}$ and $x \in A_{d}$ we have $(\sigma \otimes \mathrm{id})(\mathrm{id} \otimes \sigma)\left(\Phi_{c}(a) \otimes x\right)=\left[\left(\mathrm{id} \otimes \Phi_{c}\right) u\right]\left(x \otimes \Phi_{c}(a)\right)\left[\left(\mathrm{id} \otimes \Phi_{c}\right) u^{*}\right]$

$$
=\left(\mathrm{id} \otimes \Phi_{c}\right)\left[u(x \otimes a) u^{*}\right]=\left(\mathrm{id} \otimes \Phi_{c}\right) \sigma(a \otimes x)
$$

and (4.13) follows.

According to (2.16) and (3.8)

$$
\begin{aligned}
& \left(\kappa_{d} \otimes \kappa_{c}\right) u=u, \\
& \left(\kappa_{d} \otimes \kappa_{c}\right) u^{*}=u^{*} .
\end{aligned}
$$

Remembering that $\kappa_{d} \otimes \kappa_{c}$ is antimultiplicative we obtain

$$
\sigma s_{d c}\left(\kappa_{d} \otimes \kappa_{c}\right) \sigma=S_{c d}\left(\kappa_{c} \otimes \kappa_{d}\right)
$$

where $s_{d c}: A_{d} \otimes A_{c} \rightarrow A_{c} \otimes A_{d}$ is the flip automorphism $\left(s_{d c}(y \otimes x)=x \otimes y\right.$ for any $x \in A_{c}$ and $\left.y \in A_{d}\right)$ and $s_{c d}=s_{d c}^{-1}$.

Combining (4.9) and (3.30) we see that

$$
\left(h_{d L} \otimes h_{c}\right) \sigma=h_{c} \otimes h_{d R} .
$$


Now we shall introduce the basic notions related to the twisted product $G=G_{c}$ $\times G_{d}$. The $C^{*}$-algebra of "all continuous, vanishing at infinity functions on $G$ " is introduced by the formula

$$
A=A_{c} \otimes A_{d} .
$$

The Pedersen ideal in $A$ will be denoted by $A_{0}$. One can easily check that $A_{0}=A_{c} \otimes_{\mathrm{alg}} A_{d 0}$. We shall also use the algebra of "all smooth functions with compact support"

$$
\tilde{\mathscr{A}}=\mathscr{A}_{c} \otimes_{\mathrm{alg}} A_{d 0} .
$$

$\tilde{\mathscr{A}}$ is dense in $A$. The comultiplication, counit and coinverse related to $G$ are introduced by the formulae (cf. (4.6)-(4.8)):

$$
\begin{gathered}
\Phi=(\mathrm{id} \otimes \sigma \otimes \mathrm{id})\left(\Phi_{c} \otimes \Phi_{d}\right), \\
e=e_{c} \otimes e_{d}, \\
\kappa=s_{d c}\left(\kappa_{d} \otimes \kappa_{c}\right) \sigma .
\end{gathered}
$$

Clearly $\Phi \in \operatorname{Mor}(A, A \otimes A)$ whereas $e$ is a proper linear multiplicative $*$-functional defined on $\tilde{\mathscr{A}}$ and $\kappa$ is a proper linear antimultiplicative bijective mapping acting on $\tilde{\mathscr{A}}$. Moreover remembering that $\Phi_{c}\left(\mathscr{A}_{c}\right) \subset \mathscr{A}_{c} \otimes_{\text {alg }} \mathscr{A}_{c}$ one can verify that $\Phi(a) \in \tilde{\mathscr{A}} \otimes_{r} \tilde{\mathscr{A}}$ for any $a \in \tilde{\mathscr{A}}$. The terminology used above is justified by the following

Theorem 4.1. The comultiplication $\Phi$, counit $e$ and coinverse $\kappa$ introduced by (4.16)-(4.18) satisfy the following equalities:

and for any $a \in \tilde{\mathscr{A}}$ :

$$
(\mathrm{id} \otimes \Phi) \Phi=(\Phi \otimes \mathrm{id}) \Phi,
$$

$$
\begin{gathered}
(e \otimes \mathrm{id}) \Phi(a)=(\mathrm{id} \otimes e) \Phi(a)=a, \\
\kappa\left(\kappa\left(a^{*}\right)^{*}\right)=a, \\
m(\kappa \otimes \mathrm{id}) \Phi(a)=e(a) I, \\
m(\mathrm{id} \otimes \kappa) \Phi(a)=e(a) I, \\
\Phi(\kappa(a))=s(\kappa \otimes \kappa) \Phi(a) .
\end{gathered}
$$

In the above relations id denotes the identity map acting on $A, s: A \otimes A \rightarrow A \otimes A$ is the flip automorphism: $s(a \otimes b)=b \otimes a(a, b \in A)$ and $m: A \otimes_{r} A \ni a \otimes b \rightarrow a b \eta A$ is the multiplication map. The unity of $M(A)$ is denoted by $I$.

Proof. Using (4.7) and taking into account the coassociativity of $\Phi_{c}$ and $\Phi_{d}$ one can easily check that

$$
\begin{aligned}
& (\mathrm{id} \otimes \Phi) \Phi=\left(\mathrm{id}_{c} \otimes \psi \otimes \mathrm{id}_{d}\right)\left(\Phi_{c} \otimes \Phi_{d}\right), \\
& (\Phi \otimes \mathrm{id}) \Phi=\left(\mathrm{id}_{c} \otimes \psi^{\prime} \otimes \mathrm{id}_{d}\right)\left(\Phi_{c} \otimes \Phi_{d}\right),
\end{aligned}
$$

where

$$
\begin{aligned}
\psi & =(\mathrm{id} \otimes \mathrm{id} \otimes \sigma)\left(\mathrm{id} \otimes \Phi_{c} \otimes \mathrm{id}\right)(\sigma \otimes \mathrm{id})\left(\mathrm{id} \otimes \Phi_{d}\right), \\
\psi^{\prime} & =(\sigma \otimes \mathrm{id} \otimes \mathrm{id})\left(\mathrm{id} \otimes \Phi_{d} \otimes \mathrm{id}\right)(\mathrm{id} \otimes \sigma)\left(\Phi_{c} \otimes \mathrm{id}\right) .
\end{aligned}
$$


On the other hand using (4.12) and (4.13) we obtain

$$
\psi=(\sigma \otimes \sigma)(\mathrm{id} \otimes \sigma \otimes \mathrm{id})\left(\Phi_{c} \otimes \Phi_{d}\right)=\psi^{\prime}
$$

and (4.19) follows. In the similar way using the relations (3.6), (3.9) $(3.12)$, corresponding relations for $G_{c}$ and (4.10)-(4.14) one can prove (4.20)-(4.23). In fact (4.19) - (4.23) become trivial when one uses the diagram notation (see Appendix A for details). Equation (4.24) follows from (4.20)-(4.23) (cf. [1]). Q.E.D.

Theorem 4.1 shows that the noncommutative space $G$ is provided with a group structure. We say that $G$ is the double group built over $G_{c}$.

Let

$$
h=h_{c} \otimes h_{d R} .
$$

Clearly $h$ is a positive functional defined on the Pedersen ideal $A_{0}$ of $A$. Using the results of Sect. 3 one can easily show that $(h \otimes \mathrm{id})$ and $(\mathrm{id} \otimes h)$ can be applied to $\Phi(a)$ for any $a \in A_{0}$. We have

Theorem 4.2. $h$ is left and right Haar measure: for any $a \in A_{0}$,

$$
\begin{aligned}
& (h \otimes \mathrm{id}) \Phi(a)=h(a) I, \\
& (\mathrm{id} \otimes h) \Phi(a)=h(a) I .
\end{aligned}
$$

Proof. The above relations follow easily from (4.15), (4.12), (4.13) and invariance properties of $h_{c}, h_{d R}$ and $h_{d L}$ (cf. (3.22) and (3.25)). See Appendix A for the details. Q.E.D.

Theorem 4.2 shows in particular that the quantum group $G$ is unimodular. In the present paper we do not touch the uniqueness of the Haar measures on noncompact quantum groups. In our opinion the uniqueness up to a positive factor (and the existence) of the left and right Haar measures will be proved within the future general theory of quantum groups.

In the remaining part of this section we investigate the structure of finitedimensional representations of $G$. We describe the remarkable $1-1$ natural correspondence between the set of representations of $G$ and the set of bicovariant bimodules [12] over $G_{c}$.

In order to simplify the exposition we shall assume that the counit $e_{c}$ defined originally on $\mathscr{A}_{c}$ admits the continuous extension to the whole $A_{c}$. Then $e_{c} \in \operatorname{Mor}\left(A_{c}, \mathbb{C}\right)$. This is the case for $G_{c}=S_{\mu} U(N)$.

Let

$$
\begin{aligned}
& p_{c}=\mathrm{id}_{A_{c}} \otimes e_{d}, \\
& p_{d}=e_{c} \otimes \mathrm{id}_{A_{d}} .
\end{aligned}
$$

Clearly the morphisms $p_{c} \in \operatorname{Mor}\left(A, A_{c}\right)$ and $p_{d} \in \operatorname{Mor}\left(A, A_{d}\right)$ correspond to embeddings $G_{c} \rightarrow G$ and $G_{d} \rightarrow G$ respectively. The following theorem shows that $G_{c}$ and $G_{d}$ are subgroups of $G$ :

Theorem 4.3. We have

$$
\begin{array}{ll}
\Phi_{c} p_{c}=\left(p_{c} \otimes p_{c}\right) \Phi, & \Phi_{d} p_{d}=\left(p_{d} \otimes p_{d}\right) \Phi, \\
e_{c} p_{c}=e, & e_{d} p_{d}=e, \\
\kappa_{c} p_{c}=p_{c} \kappa, & \kappa_{d} p_{d}=p_{d} \kappa .
\end{array}
$$


Proof. The above relations follow immediately from definitions (4.16)-(4.18) and formulae (4.10) and (4.11). Q.E.D.

It follows immediately from (3.6), the corresponding relation for $G_{c}$ and definition (4.16) that

$$
\begin{aligned}
& \left(p_{c} \otimes p_{d}\right) \Phi=\mathrm{id}, \\
& \left(p_{d} \otimes p_{c}\right) \Phi=\sigma .
\end{aligned}
$$

We recall that $v$ is a representation of $G$ acting on a finite-dimensional complex vector space $K$ if $v \eta B(K) \otimes A$ and

$$
\begin{aligned}
(\mathrm{id} \otimes e) v & =I_{B(K)}, \\
(\mathrm{id} \otimes \Phi) v & =v(1) .
\end{aligned}
$$

In the similar way one defines representations of $G_{c}$ (cf. [10]) and $G_{d}$.

Theorem 4.4. Let $K$ be a finite-dimensional complex vector space, $v \eta B(K) \otimes A$, $v_{c}=\left(\mathrm{id} \otimes p_{c}\right) v$ and $v_{d}=\left(\mathrm{id} \otimes p_{d}\right) v$. Assume that $v$ is a representation of $G$. Then $v_{c}$ and $v_{d}$ are representations of $G_{c}$ and $G_{d}$ respectively,

and

$$
v=v_{c}\left(v_{d}\right.
$$

$$
v_{d}\left(v_{c}=(\mathrm{id} \otimes \sigma)\left(v_{c}(1) v_{d}\right) .\right.
$$

Conversely if $v_{c}$ and $v_{d}$ are representations of $G_{c}$ and $G_{d}$ respectively acting on $K$ satisfying relation (4.31) then $v$ introduced by (4.30) is a representation of $G$, $v_{c}=\left(\mathrm{id} \otimes p_{c}\right) v$ and $v_{d}=\left(\mathrm{id} \otimes p_{d}\right) v$.

Proof. If $v$ is a representation of $G$ then using Theorem 4.3 we see that $v_{c}$ and $v_{d}$ are representations of $G_{c}$ and $G_{d}$ respectively. Moreover applying $\left(\mathrm{id} \otimes p_{c} \otimes p_{d}\right)$ to both sides of (4.29) and using (4.27) we obtain (4.30). Similarly applying (id $\otimes p_{d} \otimes p_{c}$ ), using (4.28) and (4.30) we get (4.31).

Assume now that $v_{c} \in B(K) \otimes A_{c}$ and $v_{d} \eta B(K) \otimes A_{d}$ are representations of $G_{c}$ and $G_{d}$ respectively and that relation (4.31) holds. Applying (id $\left.\otimes \Phi\right)$ to both sides of (4.30) we obtain (cf. (4.16))

$$
\begin{aligned}
& (\mathrm{id} \otimes \Phi) v=\left(\mathrm{id} \otimes \mathrm{id}_{A_{c}} \otimes \sigma \otimes \mathrm{id}_{A_{d}}\right)\left(\mathrm{id} \otimes \Phi_{c} \otimes \Phi_{d}\right)\left(v_{c} \oplus v_{d}\right) \\
& =\left(\mathrm{id} \otimes \mathrm{id}_{A_{c}} \otimes \sigma \otimes \mathrm{id}_{A_{d}}\right)\left(v _ { c } \oplus v _ { c } \left(v_{d}\left(v_{d}\right)\right.\right. \\
& =v_{c}\left(v _ { d } \left(v_{c} \oplus v_{d}=v(v)\right.\right. \text {. }
\end{aligned}
$$

In the next to the last step we used (4.31). It shows that $v$ is a representation of $G$. The remaining relations are obvious. Q.E.D.

In practical cases (see the next section) the compatibility (4.31) may be difficult to prove. We shall formulate this condition in a form easy to verify. Let $\xi$ be a linear mapping from $\mathscr{A}_{c}$ into $B(K)$. For any $a \in \mathscr{A}_{c}$ we set

$$
\begin{aligned}
& \xi * a=\sum_{i} m^{i} \otimes\left(\xi_{i} * a\right), \\
& a * \xi=\sum_{i} m^{i} \otimes\left(a * \xi_{i}\right),
\end{aligned}
$$


where $m^{i} \in B(K)$ and $\xi_{i} \in \mathscr{A}_{c}^{\prime}$ are related to $\xi$ by formula (3.33). Clearly $\xi * a$, $a * \xi \in B(K) \otimes \mathscr{A}_{c}$.

Proposition 4.5. Let $v_{c}$ and $v_{d}$ be representations of $G_{c}$ and $G_{d}$ acting on $K$ and $\xi$ be the linear multiplicative mapping from $\mathscr{A}_{c}$ into $B(K)$ related to $v_{d}$ via formulae (3.31)-(3.33).

Then condition (4.31) holds if and only if

$$
(a * \xi) v_{c}=v_{c}(\xi * a)
$$

for any $a \in \mathscr{A}_{c}$.

Remark. Verifying (4.32) it is sufficient (due to the multiplicativity of $\xi$ ) to check it for generators of the algebra $\mathscr{A}_{c}$. For example if $G_{c}=S_{\mu} U(2)$ then it is sufficient to consider $a=\alpha, \gamma, \alpha^{*}$, and $\gamma^{*}$.

Proof. Let $\zeta$ be any proper linear functional on $A_{d}$ and

$$
a=(\zeta \otimes \mathrm{id}) u^{*} \text {. }
$$

Clearly $a \in \mathscr{A}_{c}$ and any element of $\mathscr{A}_{c}$ is of this form. Using formulae (2.15) and (3.31) (3.33) one can easily check that (leg numbering notation!)

$$
\begin{aligned}
& \xi * a=(\mathrm{id} \otimes \zeta \otimes \mathrm{id}) v_{d 12} u_{23}^{*}, \\
& a * \xi=(\mathrm{id} \otimes \zeta \otimes \mathrm{id}) u_{23}^{*} v_{d 12} .
\end{aligned}
$$

Assume that (4.31) holds. Taking into account (4.9) we have

$$
v_{d 12} v_{c 13}=(\mathrm{id} \otimes \sigma)\left(v_{c 12} v_{d 13}\right)=u_{23} v_{c 13} v_{d 12} u_{23}^{*} \text {. }
$$

Therefore

$$
u_{23}^{*} v_{d 12} v_{c 13}=v_{c 13} v_{d 12} u_{23}^{*}
$$

Applying (id $\otimes \zeta \otimes \mathrm{id})$ to both sides of the above relation and taking into account (4.33) and (4.34) we obtain (4.32). Conversely if (4.32) holds then reading the above formulae in reverse order we get (4.31). Q.E.D.

The remaining part of this section heavily depends on concepts and results contained in [12]. We shall use letters "DC" (means Differential Calculus) to indicate references to formulae and theorems contained in that paper.

Theorem 4.6. Let $v$ be a finite-dimensional representation of $G$. Then there exists a bicovariant $\mathscr{A}_{c}$-bimodule $\left(\Gamma, \Phi_{\Gamma, \Gamma} \Phi\right)$ such that

$1^{\circ}$ The space ${ }_{\mathrm{inv}} \Gamma$ of left-invariant elements of $\Gamma$ coincides with the carrier space of $v$.

$2^{\circ}$ For any $a \in \mathscr{A}_{c}$ and $\omega \in_{\mathrm{inv}} \Gamma$ we have

$$
\begin{aligned}
& a \omega=\sum_{i}\left(m^{i} \omega\right)\left(\xi_{i} * a\right), \\
& \Gamma \Phi \omega=\sum_{j}\left(n^{j} \omega\right) \otimes v_{c j},
\end{aligned}
$$


where $m^{i}, n^{j} \in B\left({ }_{\mathrm{inv}} \Gamma\right), \xi_{i} \in \mathscr{A}_{c}^{\prime}$ and $v_{c j} \in \mathscr{A}_{c}$ are related to $v$ in the following way:

$$
\begin{aligned}
& \left(\mathrm{id} \otimes p_{c}\right) v=\sum_{j} n^{j} \otimes v_{c j}, \\
& \left(\mathrm{id} \otimes p_{d}\right) v=\sum_{i} m^{i} \otimes\left(\mathrm{id} \otimes \xi_{i}\right) u^{*} .
\end{aligned}
$$

Any bicovariant $\mathscr{A}_{c}$-bimodule such that the space of left-invariant elements is finite-dimensional arises in this way. The correspondence

$$
\text { representations } \leftrightarrow \text { bimodules }
$$

is bijective and natural in the following sense:

$1^{\circ}$ If $v^{1}$ and $v^{2}$ are finite-dimensional representations of $G$ and $\Gamma^{1}$ and $\Gamma^{2}$ are corresponding bimodules then any operator intertwining $v^{1}$ and $v^{2}$ uniquely extends to a bicovariant bimodule homomorphism $\Gamma^{1} \rightarrow \Gamma^{2}$. Conversely any bicovariant bimodule homomorphism $\Gamma^{1} \rightarrow \Gamma^{2}$ restricted to left-invariant elements intertwines $v^{1}$ with $v^{2}$.

$2^{\circ}$ Tensor product of representations corresponds to the tensor product of corresponding bimodules.

$3^{\circ}$ Complex conjugate representation corresponds to the complex conjugate bimodule.

Proof. We will indicate the main points only. Let $\omega_{1}, \omega_{2}, \ldots, \omega_{N}$ be the basis in ${ }_{\text {inv }} \Gamma$ and $\left(m_{k l}^{i}\right)$ and $\left(n_{k l}^{j}\right)$ be matrices corresponding to $m^{i}$ and $n^{j}$ :

We set

$$
\begin{aligned}
& m^{i} \omega_{l}=\sum_{k} m_{k l}^{i} \omega_{k}, \\
& n^{j} \omega_{l}=\sum_{j} n_{k l}^{j} \omega_{k} .
\end{aligned}
$$

$$
\begin{aligned}
f_{k l} & =\sum_{i} m_{l k}^{i}\left(\xi_{i} \circ \kappa_{c}\right), \\
R_{k l} & =\sum_{j} n_{k l}^{j} v_{c j},
\end{aligned}
$$

$k, l=1,2, \ldots, N$. Then (4.35) and (4.36) coincides with (DC 2.14) and (DC 2.35). One can check that the assumptions of Theorem DC 2.5 are satisfied: (DC 2.15) and (DC 2.16) follow from the multiplicativity of $\xi$, (DC 2.36) and (DC 2.37) mean that (4.37) is a representation of $G_{c}$ and (DC 2.39) is equivalent to (4.32). It proves the existence of the desired bimodule. The remaining details are left to the reader. Q.E.D.

Remark. In May 1987 after a seminar in IHES (Bures sur Yvette) devoted to differential calculi on quantum groups Connes conjectured that bicovariant bimodules over the algebra of "smooth functions" on a quantum group are (in natural way) labeled by representations of another quantum group. He proposed the name "structure group" for the latter group. We tested this idea for $S_{\mu} U(2)$ group arriving in the summer of 1987 to the commutation relations (1.9)-(1.25). Next we found the complete set of irreducible representations for these relations. Analysing the representations we discovered in March 1989 the Iwasawa 
decomposition. This result suggested that the structure group is a combination of the original group and its Pontryagin dual. At this moment we started to analyse the formula (DC 2.39) which seems to be the most important relation of the theory of bicovariant bimodules. Unfortunately the indexes labeling the functionals $f_{k l}$ in this formula are rather related to the basis contragradient to $\left(\omega_{k}\right)$ then to $\left(\omega_{k}\right)$ itself. This is why the position of summation indexes in (DC 2.39) is strange. Using consequently the indexes related to $\left(\omega_{k}\right)$ (cf. (4.16) where the order of indexes $k, l$ on the right-hand side is opposite to that on the left one) we arrived at the compatibility condition (4.31) with $\sigma$ introduced by (4.9). The definition (4.9) is in turn the key formula in the present paper.

In our analysis of finite-dimensional representations of $G$ we assumed that $e_{c}$ is norm continuous. If this is not the case then in Theorem 4.4 and 4.6 one has to assume additionally that the matrix elements of $v$ belong to $\tilde{\mathscr{A}}$.

\section{Double Group Built over $S_{\mu} U(2)$}

In this section we apply the general theory developed in Sects. 2, 3, 4 to the quantum group $S_{\mu} U(2)$ introduced in [9]. The Pontryagin dual and the double group will be described in detail. In particular we shall construct a twodimensional representation $w$ of the double group obeying all the properties discussed in Sect.1. In a certain sense (see Theorem 5.4) the algebra of all continuous vanishing at infinity functions on the double group is generated by matrix elements of $w$. These results allow us to identify the double group built over $S_{\mu} U(2)$ with the quantum Lorentz group introduced in Sect. 1.

In the remaining part of the paper $G_{c}=S_{\mu} U(2), G_{d}$ is the Pontryagin dual of $S_{\mu} U(2)$ and $G$ denotes the corresponding double group. Consequently $A_{c}, \Phi_{c}, \kappa_{c}, \ldots$ $\left(A_{d}, \Phi_{d}, \kappa_{d}, \ldots\right.$, and $A, \Phi, \kappa, \ldots$ respectively) will denote the objects related to $S_{\mu} U(2)$ (the Pontryagin dual of $S_{\mu} U(2)$ and the double group built over $S_{\mu} U(2)$ respectively). We shall assume that $|\mu|<1$ and $\mu \neq 0$.

Let $\xi: \mathscr{A}_{c} \rightarrow M_{2}(\mathbb{C})$ be the linear multiplicative mapping such that

$$
\begin{aligned}
\xi\left(\alpha_{c}\right)=\left(\begin{array}{cc}
\mu^{1 / 2}, & 0 \\
0, & \mu^{-1 / 2}
\end{array}\right), & \xi\left(\gamma_{c}^{*}\right)=\left(\begin{array}{cc}
0, & 0 \\
0, & 0
\end{array}\right), \\
\xi\left(\gamma_{c}\right)=\left(\begin{array}{cc}
0, & \mu^{-3 / 2}\left(\mu^{2}-1\right) \\
0, & 0
\end{array}\right), & \xi\left(\alpha_{c}^{*}\right)=\left(\begin{array}{cc}
\mu^{-1 / 2}, & 0 \\
0, & \mu^{1 / 2}
\end{array}\right) .
\end{aligned}
$$

To prove the existence of $\xi$ it is sufficient to check that the matrices standing on the right-hand sides of (5.1) satisfy the commutation relations characteristic for generators of $\mathscr{A}_{c}$. The easy exercise is left to the reader.

Let $\xi_{i j}(i, j=1,2)$ be linear functionals on $\mathscr{A}_{c}$ such that

$$
\xi(x)=\left(\xi_{i j}(x)\right)_{i, j=1,2}
$$

for any $x \in \mathscr{A}_{c}$. According to (5.1),

$$
\begin{aligned}
& \left.\xi_{11}\left(\alpha_{c}\right)=\mu^{1 / 2}, \quad \xi_{11}\left(\alpha_{c}^{*}\right)=\mu^{-1 / 2}, \quad \xi_{12}\left(\gamma_{c}\right)=\mu^{-3 / 2}\left(\mu^{2}-1\right),\right\} . \\
& \left.\xi_{22}\left(\alpha_{c}\right)=\mu^{-1 / 2}, \quad \xi_{22}\left(\alpha_{c}^{*}\right)=\mu^{1 / 2}, \quad \xi_{11}(I)=1, \quad \xi_{22}(I)=1\right\}
\end{aligned}
$$

All other $\xi_{i j}(x)\left(i, j=1,2 ; x=I, \alpha_{c}, \gamma_{c}, \alpha_{c}^{*}, \gamma_{c}^{*}\right)$ vanish. 
The structure of the Pontryagin dual of $S_{\mu} U(2)($ for $|\mu|<1)$ is described in detail by the following

Theorem 5.1. Let $w_{d}$ be the representation of $G_{d}$ corresponding to $\xi$ via formulae (3.31)-(3.33). Then

$$
w_{d}=\left(\begin{array}{cc}
a, & n \\
0, & a^{-1}
\end{array}\right),
$$

where a, $n \eta A_{d}$ satisfy relations (1.35)-(1.38) and $S p a \subset S_{\mu}$.

$2^{\circ}$ The comultiplication, counit and coinverse act in the following way:

$$
\begin{gathered}
\Phi_{d}(a)=a \otimes a, \quad \Phi_{d}(n)=a \otimes n+n \otimes a^{-1}, \\
e_{d}(a)=1, \quad e_{d}(n)=0, \\
\kappa_{d}(a)=a^{-1}, \quad \kappa_{d}(n)=-\mu^{-1} n, \\
\kappa_{d}\left(a^{*}\right)=\left(a^{-1}\right)^{*}, \quad \kappa_{d}\left(n^{*}\right)=-\mu n^{*} .
\end{gathered}
$$

$3^{\circ}$ Elements $a$ and $n$ generate $A_{d}$ in the following sense:

For any bounded operators $\tilde{a}, \tilde{n}$ acting on a Hilbert space $H$ such that $\tilde{a}$ is invertible, $S p \tilde{a} \subset S_{\mu}$ and the relations (1.35)-(1.38) with a, $n$ replaced by $\tilde{a}, \tilde{n}$ are satisfied there exists unique nondegenerate representation $\pi$ of $A_{d}$ acting on $H$ such that $\tilde{a}=\pi(a)$ and $\tilde{n}=\pi(n)$.

Proof. Ad $1^{\circ}$. In the considered case the decompositions (2.11) and (2.14) assume the following form:

$$
\begin{gathered}
A_{d}=\sum_{s}^{\oplus} B\left(H^{s}\right), \\
u=\sum_{s}^{\oplus} u^{s},
\end{gathered}
$$

where $u^{s}$ is the irreducible $(2 s+1)$-dimensional unitary representation of $G_{c}=S_{\mu} U(2)$ acting on the Hilbert space $H^{s}$ and $s$ runs over the set of all nonnegative integers and half-integers. In what follows $\pi_{s}$ denotes the canonical projection $A_{d} \rightarrow B\left(H^{s}\right)$. Let

$$
w_{d}=\sum_{s}^{\oplus} w_{d}^{s}
$$

be the corresponding decomposition of $w_{d}$ : each $w_{d}^{s}=\left(\mathrm{id} \otimes \pi_{s}\right) w_{d}$ is a $2 \times 2$-matrix with entries belonging to $B\left(H^{s}\right)$. According to (3.31)-(3.33)

$$
w_{d}^{s}=\left(\left(\mathrm{id} \otimes \xi_{i j}\right) u^{s *}\right)_{i, j=1,2} .
$$

Statement $1^{\circ}$ of the theorem asserts that all $w_{d}^{s}(s=0,1 / 2,1, \ldots)$ are $A N_{\mu^{-}}$ matrices. We shall use induction with respect to $s$.

For $s=0$ the statement is trivial. For $s=1 / 2$, remembering that

$$
u^{1 / 2}=\left(\begin{array}{cc}
\alpha_{c}, & -\mu \gamma_{c}^{*} \\
\gamma_{c}, & \alpha_{c}^{*}
\end{array}\right),
$$


and using (5.2) we obtain

$$
w_{d}^{1 / 2}=\left[\begin{array}{cc}
\left(\begin{array}{cc}
\mu^{-1 / 2}, & 0 \\
0, & \mu^{1 / 2}
\end{array}\right), & \left(\begin{array}{cc}
0, & 0 \\
\mu^{-1 / 2}\left(1-\mu^{2}\right), & 0
\end{array}\right) \\
\left(\begin{array}{ll}
0, & 0 \\
0, & 0
\end{array}\right), & \left(\begin{array}{cc}
\mu^{1 / 2}, & 0 \\
0, & \mu^{-1 / 2}
\end{array}\right)
\end{array}\right]
$$

and elementary computations show that $w_{d}^{1 / 2}$ is an $A N_{\mu}$-matrix.

Assume now that $w_{d}^{s}$ is an $A N_{\mu}$-matrix. We know that $w_{d}$ is a representation of $G_{d}$ :

$$
\left(\mathrm{id} \otimes \Phi_{d}\right) w_{d}=w_{d}(1) w_{d} .
$$

Applying (id $\left.\otimes \pi_{1 / 2} \otimes \pi_{s}\right)$ to both sides of this relation we get

$$
\left[\operatorname{id} \otimes\left(\pi_{1 / 2} \otimes \pi_{s}\right) \Phi_{d}\right] w_{d}=w_{d}^{1 / 2}(1) w_{d}^{s},
$$

and using Proposition 1.2 we see that

$$
\left[\mathrm{id} \otimes\left(\pi_{1 / 2} \otimes \pi_{s}\right) \Phi_{d}\right] w_{d}
$$

is an $A N_{\mu}$-matrix. According to Proposition $3.2\left(\pi_{1 / 2} \otimes \pi_{s}\right) \circ \Phi_{d}$ restricted to $B\left(H^{s+1 / 2}\right)$ is an embedding (spin $(s+1 / 2)$ representation of $S_{\mu} U(2)$ is contained in the tensor product of spin $1 / 2$ and spin $s$ representations). Therefore $w_{d}^{s+1 / 2}$ is an $A N_{\mu}$-matrix and the proof of Statement $1^{\circ}$ is complete.

$\operatorname{Ad} 2^{\circ}$. (5.3) is a representation of $G_{d}$ and relations (5.4)-(5.6) follow immediately from this fact. Using (5.6) and remembering that $\kappa_{d}$ followed by $*$ is an involution we obtain (5.7).

Ad $3^{\circ}$. According to (5.8) and (5.9),

and

$$
\pi_{1 / 2}(a)=\left(\begin{array}{cc}
\mu^{-1 / 2}, & 0 \\
0, & \mu^{1 / 2}
\end{array}\right)
$$

$$
\left(\pi_{1 / 2} \otimes \pi_{s}\right) \Phi_{d}(a)=\pi_{1 / 2}(a) \otimes \pi_{s}(a) .
$$

We also know (cf. Proposition 3.2) that $\left(\pi_{1 / 2} \otimes \pi_{s}\right) \Phi_{d}$ restricted to $B\left(H^{s+1 / 2}\right)$ and $B\left(H^{s-1 / 2}\right)$ are embeddings. Taking into account these facts and using the mathematical induction one can easily show that

$$
\operatorname{Sp} \pi_{s}(a)=\left\{\mu^{-s}, \mu^{-s+1}, \ldots, \mu^{s}\right\} .
$$

A detailed analysis of the commutation relations (1.35)-(1.38) shows that if $(\tilde{a}, \tilde{n})$ is a pair of bounded operators acting in a irreducible way on a Hilbert space $H$ satisfying these relations and condition $S p \tilde{a} \subset S_{\mu}$ then $H$ is finite-dimensional. For each $s=0,1 / 2,1, \ldots$ there exists one and only one (up to unitary equivalence) such a pair $\left(\tilde{a}_{s}, \tilde{n}_{s}\right)$ acting on $(2 s+1)$-dimensional Hilbert space: There exists an orthonormal basis $\{|m, s\rangle: m=-s,-s+1, \ldots, s\}$ such that

$$
\begin{aligned}
& \tilde{a}_{s}|m, s\rangle=\mu^{m}|m, s\rangle, \\
& \tilde{n}_{s}|m, s\rangle=\sqrt{|\mu|^{-2 s}-|\mu|^{-2 m}-|\mu|^{2 m+2}+|\mu|^{2 s+2}}|m+1, s\rangle .
\end{aligned}
$$

In particular $S p \tilde{a}_{s}$ coincides with (5.10). 
Assume for the moment that the pair $\left(\pi_{s}(a), \pi_{s}(n)\right)$ is reducible. Then passing to an irreducible subspace we would find a pair $(\tilde{a}, \tilde{n})$ satisfying the all considered requirements, acting on a Hilbert space of dimension smaller than $2 s+1$ and such that $S p \tilde{a}$ contains $\mu^{s}$. According to the above mentioned analysis such a pair does not exist. Therefore $\left(\pi_{s}(a), \pi_{s}(n)\right)$ is irreducible, $\left(\tilde{a}_{s}, \tilde{n}_{s}\right)$ must be equivalent to $\left(\pi_{s}(a)\right.$, $\left.\pi_{s}(n)\right)$ and Statement $3^{\circ}$ follows. Q.E.D.

Corollary 5.2. There exists an orthonormal basis $\{|m, s\rangle: m=-s,-s+1, \ldots, s\}$ in $H^{s}$ such that

$$
\begin{aligned}
& \pi_{s}(a)|m, s\rangle=\mu^{m}|m, s\rangle, \\
& \pi_{s}(n)|m, s\rangle=\sqrt{|\mu|^{-2 s}-|\mu|^{-2 m}-|\mu|^{2 m+2}+|\mu|^{2 s+2}}|m+1, s\rangle .
\end{aligned}
$$

Let $\mathscr{A}_{d}$ be the $*$-algebra of elements affiliated with $A_{d}$ generated by $a, a^{-1}, n$. We set $c=a^{*} a^{-1}$. According to $(5.11) \pi_{s}(c)=(\operatorname{sign} \mu)^{2 s} I_{s}$ for all s. Hence $c=c^{*}$ is in the center of $\mathscr{A}_{d}$ and $c^{2}=I_{d}$. For $\mu>0, c=I_{d}$.

Proposition 5.3. The elements

$$
c^{k} a^{l} n^{r} n^{* t}
$$

where $k=0,1$ ( for $\mu>0, k=0$ ), l-integer, $r, t=0,1,2, \ldots$ form a linear basis in $\mathscr{A}_{d}$. Proof. According to (1.35)-(1.38) any element of $\mathscr{A}_{d}$ is a finite linear combination of elements (5.12). To end the proof it suffices to show that these elements are linearly independent.

Assume that

$$
Q=\sum q_{k l r t} c^{k} a^{l} n^{r} n^{* t}
$$

is a nontrivial linear combination of elements (5.12). We have to show that

$$
Q \neq 0 \text {. }
$$

To this end we choose $k_{0}, l_{0}, r_{0}$, and $t_{0}$ such that

$$
\begin{aligned}
q_{k_{0} l_{0} r_{0} t_{0}} & \neq 0, \\
q_{k l r t} & =0
\end{aligned}
$$

for all $k, l, r, t$ such that $r+t>r_{0}+t_{0}$ and

$$
q_{k l r_{0} t_{0}}=0
$$

for all $k, l$ such that $l>l_{0}$. In the following $\lim _{s \rightarrow \infty}^{+}\left(\lim _{s \rightarrow \infty}^{-}\right.$respectively $)$denotes $\lim _{s \rightarrow \infty}$, where $s$ runs over integers (half-integers respectively). Using (5.11) we get

$$
\begin{gathered}
\lim _{m \rightarrow \infty} \lim _{s \rightarrow \infty}^{ \pm}\left\langle m, s\left|\pi_{s}(Q)\right| m+t_{0}-r_{0}, s\right\rangle|\mu|^{s\left(r_{0}+t_{0}\right)} \mu^{-m l_{0}} \\
\quad=\left\{\begin{array}{lll}
q_{0 l_{0} r_{0} t_{0}} \pm q_{1 l_{0} r_{0} t_{0}} & \text { for } & \mu<0 \\
q_{0 l_{0} r_{0} t_{0}} & \text { for } & \mu>0
\end{array}\right.
\end{gathered}
$$

and (cf. (5.14)) relation (5.13) follows. Q.E.D. 
Now we are able to give the detailed description of the double group built over $S_{\mu} U(2)$ (for $\left.|\mu|<1\right)$.

Theorem 5.4. Let us consider the following four elements affiliated with $A=A_{c} \otimes A_{d}:$

$$
\left.\begin{array}{l}
\alpha=\alpha_{c} \otimes a, \\
\beta=\alpha_{c} \otimes n-\mu \gamma_{c}^{*} \otimes a^{-1}, \\
\gamma=\gamma_{c} \otimes a, \\
\delta=\gamma_{c} \otimes n+\alpha_{c}^{*} \otimes a^{-1} .
\end{array}\right\}
$$

Then

$1^{\circ} \alpha, \beta, \gamma, \delta$ satisfy relations (1.9)-(1.25).

$2^{\circ}$ The matrix

$$
w=\left(\begin{array}{ll}
\alpha, & \beta \\
\gamma, & \delta
\end{array}\right)
$$

is a representation of $G$.

$3^{\circ}$ The comultiplication, counit and coinverse act in the following way:

$$
\begin{aligned}
& \Phi(\alpha)=\alpha \otimes \alpha+\beta \otimes \gamma, \\
& \Phi(\beta)=\alpha \otimes \beta+\beta \otimes \delta, \\
& \Phi(\gamma)=\gamma \otimes \alpha+\delta \otimes \gamma, \\
& \Phi(\delta)=\gamma \otimes \beta+\delta \otimes \delta, \\
& e(\alpha)=e(\delta)=1, \quad e(\beta)=e(\gamma)=0, \\
& \kappa(\alpha)=\delta, \quad \kappa(\beta)=-\mu^{-1} \beta, \quad \kappa(\gamma)=-\mu \gamma, \quad \kappa(\delta)=\alpha, \\
& \kappa\left(\alpha^{*}\right)=\delta^{*}, \quad \kappa\left(\beta^{*}\right)=-\mu \beta^{*}, \quad \kappa\left(\gamma^{*}\right)=-\mu^{-1} \gamma^{*}, \quad \kappa\left(\delta^{*}\right)=\alpha^{*} .
\end{aligned}
$$

$4^{\circ}$ Elements $\alpha, \beta, \gamma$, and $\delta$ generate $A$ in the following sense:

For any bounded operators $\tilde{\alpha}, \widetilde{\beta}, \tilde{\gamma}, \tilde{\delta}$ acting on a Hilbert space $H$ and satisfying the relations (1.9)-(1.25) with $\alpha, \beta, \gamma, \delta$ replaced by $\tilde{\alpha}, \widetilde{\beta}, \tilde{\gamma}, \tilde{\delta}$ there exists a unique representation $\pi$ of $A$ acting on $H$ such that $\tilde{\alpha}=\pi(\alpha), \widetilde{\beta}=\pi(\beta), \tilde{\gamma}=\pi(\gamma)$, and $\tilde{\delta}=\pi(\delta)$.

Proof.

Ad $1^{\circ}$. Using the known commutation relations satisfied by $\alpha_{c}$ and $\gamma_{c}$ (cf. (1.29)-(1.33)) and by $a$ and $n$ (cf. (1.35)-(1.38)) one can verify that elements (5.15) satisfy relations (1.9) (1.25) (cf. the proof of Proposition 1.1).

Ad $2^{\circ}$. Relations (5.15) mean that

$$
w=w_{c}(1) w_{d} .
$$

Therefore (cf. Theorem 4.4 and Proposition 4.5) it is sufficient to show that

$$
(x * \xi) w_{c}=w_{c}(\xi * x)
$$

for $x=\alpha_{c}, \gamma_{c}, \alpha_{c}^{*}$, and $\gamma_{c}^{*}$. By easy computation this relation is equivalent to

$$
\left(w_{c} \odot w_{c}\right)(\lambda \otimes I)=(\lambda \otimes I)\left(w_{c} \odot w_{c}\right),
$$


where $\lambda: \mathbb{C}^{2} \otimes \mathbb{C}^{2} \rightarrow \mathbb{C}^{2} \otimes \mathbb{C}^{2}$ is the linear mapping defined by

$$
\lambda\left(\varepsilon_{i} \otimes \varepsilon_{j}\right)=\sum_{k, l=1}^{2} \xi_{k j}\left(w_{c l i}\right) \varepsilon_{k} \otimes \varepsilon_{l},
$$

where $i, j=1,2 ;\left(\varepsilon_{1}, \varepsilon_{2}\right)$ is the canonical basis in $\mathbb{C}^{2}$ and $w_{c l i}$ are matrix elements of $w_{c}$.

Using (5.2) one can check that

$$
\lambda=\mu^{-1 / 2} I-\mu^{-3 / 2} E \cdot E^{*}
$$

( $E$ is introduced in Sect. 1) and (5.18) follows.

Ad $3^{\circ}$. Like in the proof of Theorem 5.1 this statement follows immediately from the fact that $w$ is a representation of $G$.

Ad $4^{\circ}$. It follows immediately from the Iwasawa decomposition (Theorem 1.3), Theorem 5.1.3 ${ }^{\circ}$ and the corresponding result for $G_{c}$ (Theorem 1.1 of [9]). Q.E.D.

Let $\mathscr{A}$ be the *-algebra of elements affiliated to $\mathscr{A}$ generated by $\alpha, \beta, \gamma$, and $\delta$. To simplify the notation for any integer $s$ we set

$$
\alpha_{s}=\left\{\begin{array}{lll}
\alpha^{s} & \text { if } & s \geqq 0 \\
\delta^{-s} & \text { if } & s<0
\end{array} .\right.
$$

Similarly

$$
\alpha_{c s}=\left\{\begin{array}{lll}
\alpha_{c}^{s} & \text { if } & s \geqq 0 \\
\left(\alpha_{c}^{*}\right)^{-s} & \text { if } & s<0
\end{array} .\right.
$$

With this notation we have (cf. [9, Theorem 1.2])

Proposition 5.5. The elements

$$
\alpha_{c s} \gamma_{c}^{n} \gamma_{c}^{* m}
$$

where s-integer and $m, n=1,2, \ldots$ form a linear basis in $\mathscr{A}_{c}$.

We end this section with the following

Proposition 5.6. The elements

$$
\alpha_{r} \beta^{s} \gamma^{t} \gamma^{* t^{\prime}} \beta^{* s^{\prime}} \alpha_{r^{\prime}}^{*}
$$

where $r, r^{\prime}$ are integers and $s, t, s^{\prime}, t^{\prime}=0,1,2, \ldots$, form a linear basis in $\mathscr{A}$.

Proof. According to (1.9)-(1.25) any element of $\mathscr{A}$ is a finite linear combination of elements (5.20). We have to show that these elements are linearly independent. By virtue of Proposition 5.3 and Proposition 5.5, the elements

$$
\alpha_{c s} \gamma_{c}^{n} \gamma_{c}^{* m} \otimes c^{k} a^{l} n^{r} n^{* t},
$$

where $s, l$-integers, $m, n, r, t=0,1,2, \ldots ; k=0,1$ (for $\mu>0, k=0$ ), are linearly independent.

According to (1.29)-(1.33) for any integers $r, r^{\prime}$ we have

$$
\alpha_{c r} \alpha_{c r^{\prime}}=\alpha_{c, r+r^{\prime}}+o\left(\left|\gamma_{c}\right|\right),
$$


where $o\left(\left|\gamma_{c}\right|\right)$ is a linear combination of elements (5.19) with $n+m>1$. Using this formula, definitions (5.15) and commutation relations (1.31)-(1.33) and (1.35) -(1.38) we obtain

$$
\alpha_{r} \beta^{s} \gamma^{t} \gamma^{* t^{\prime}} \beta^{* s^{\prime}} \alpha_{r^{\prime}}^{*}=\mu^{Q} \alpha_{c, r+s-r^{\prime}-s^{\prime}} \gamma_{c}^{t} \gamma_{c}^{* t^{\prime}} \otimes c^{t^{\prime}+r^{\prime}} a^{r+t+r^{\prime}+t^{\prime}} n^{s} n^{* s^{\prime}}+o\left(\left|\gamma_{c}\right|^{t+t^{\prime}}\right),
$$

where $Q=\left(r^{\prime}+s^{\prime}-s\right)\left(t+t^{\prime}\right)+r^{\prime}\left(s^{\prime}-s\right)$ and $o\left(\left|\gamma_{c}\right|^{t+t^{\prime}}\right)$ is a linear combination of elements (5.21) with $n+m>t+t^{\prime}$. Due to this formula the linear independence of (5.20) follows easily from that of (5.21). Q.E.D.

\section{Finite-Dimensional Representations and the Groups Related to $S_{\mu} L(2, \mathbb{C})$}

In this section we investigate finite-dimensional representations of $G=S_{\mu} L(2, \mathbb{C})$. We shall prove that the theory of smooth representations is the same as that of the classical Lorentz group. There exist however non-smooth finite-dimensional representations.

Let $\mathscr{A}_{\text {hol }}$ be the subalgebra of $\mathscr{A}$ generated by $\alpha, \beta, \gamma$, and $\delta$. We say that a finitedimensional representation $v$ of $G$ is smooth (holomorphic respectively) if the matrix elements of $v$ belong to $\mathscr{A}\left(\mathscr{A}_{\text {hol }}\right.$ respectively). It follows immediately from (5.17) that $\Phi(\mathscr{A}) \subset \mathscr{A} \otimes_{\mathrm{alg}} \mathscr{A}$ and

$$
\Phi\left(\mathscr{A}_{\text {hol }}\right) \subset \mathscr{A}_{\text {hol }} \otimes_{\text {alg }} \mathscr{A}_{\text {hol }} \text {. }
$$

Using Proposition 5.6 one can easily prove

\section{Proposition 6.1.}

$1^{\circ}$ There exists linear multiplicative bijection

$$
q: \mathscr{A}_{\mathrm{hol}} \rightarrow \mathscr{A}_{c}
$$

such that

$$
\begin{gathered}
q(\alpha)=\alpha_{c}, \quad q(\beta)=-\mu \gamma_{c}^{*}, \\
q(\gamma)=\gamma_{c}, \quad q(\delta)=\alpha_{c}^{*} .
\end{gathered}
$$

$2^{\circ}$ There exists linear bijection

$$
m: \mathscr{A}_{\mathrm{hol}} \otimes_{\mathrm{alg}} \mathscr{A}_{\mathrm{hol}}^{*} \rightarrow \mathscr{A}
$$

such that

$$
m\left(x \otimes y^{*}\right)=x y^{*}
$$

for any $x, y \in \mathscr{A}_{\mathrm{hol}}$.

Let us notice that $q$ is an isomorphism in the sense of Hopf-algebra theory, i.e.

$$
\begin{aligned}
\Phi_{c} \circ q(x) & =(q \otimes q) \Phi(x), \\
e_{c} \circ q(x) & =e(x)
\end{aligned}
$$

for any $x \in \mathscr{A}_{\text {hol }}$. Indeed one can easily verify that these relations hold for $x=\alpha, \beta, \gamma, \delta$ and that the involved expressions are multiplicative with respect to $x$. 
Having in mind Proposition 6.1 one can construct linear mappings

$$
p, \bar{p}: \mathscr{A} \rightarrow \mathscr{A}_{c}
$$

such that

$$
\begin{aligned}
& p\left(x y^{*}\right)=q(x) \overline{e(y)}, \\
& \bar{p}\left(x y^{*}\right)=e(x) q(y)^{*}
\end{aligned}
$$

for any $x, y \in \mathscr{A}_{\mathrm{hol}}$. By virtue of (6.3),

$$
\left.\begin{array}{c}
\Phi_{c} \circ p(x)=(p \otimes p) \Phi(x), \\
\Phi_{c} \circ \bar{p}(x)=(\bar{p} \otimes \bar{p}) \Phi(x), \\
p(x)=e(x), \quad e_{c} \circ \bar{p}(x)=e(x)
\end{array}\right\}
$$

for any $x \in \mathscr{A}$. Moreover, using (4.20) and (6.1), for any $x, y \in \mathscr{A}_{\text {hol }}$ we get

$$
\begin{aligned}
& (p \otimes \bar{p}) \circ \Phi\left(x y^{*}\right)=q(x) \otimes q(y)^{*}, \\
& (\bar{p} \otimes p) \circ \Phi\left(x y^{*}\right)=q(y)^{*} \otimes q(x) .
\end{aligned}
$$

Comparing these two relations we obtain

$$
s_{c c}(\bar{p} \otimes p) \Phi(x)=(p \otimes \bar{p}) \Phi(x)
$$

for any $x \in \mathscr{A}\left(s_{c c}\right.$ denotes the flip automorphism of $\left.A_{c} \otimes A_{c}\right)$. Relation (6.6) shows also that the composition $(p \otimes \bar{p}) \circ \Phi$ is an invertible linear mapping acting from $\mathscr{A}$ onto $\mathscr{A}_{c} \otimes_{\text {alg }} \mathscr{A}_{c}$. The inverse of this mapping will be denoted by $\psi$ :

$$
\psi\left(x \otimes y^{*}\right)=q^{-1}(x) q^{-1}(y)^{*}
$$

for any $x, y \in \mathscr{A}_{c}$. Using this formula one can check that

$$
\begin{gathered}
\Phi \circ \psi(x \otimes y)=(\psi \otimes \psi)\left(\mathrm{id} \otimes s_{c c} \otimes \mathrm{id}\right)\left(\Phi_{c} \otimes \Phi_{c}\right)(x \otimes y), \\
p \psi(x \otimes y)=x e_{c}(y), \\
\bar{p} \psi(x \otimes y)=e_{c}(x) y
\end{gathered}
$$

for any $x, y \in \mathscr{A}_{c}$.

The following Proposition shows that the classification of finite-dimensional smooth representations of $S_{\mu} L(2, \mathbb{C})$ is equivalent to that of pairs of commuting representations of $S_{\mu} U(2)$.

Proposition 6.2. Let $K$ be a finite-dimensional complex vector space, $v \in B(K) \otimes \mathscr{A}$, $v_{1}=(\mathrm{id} \otimes p) v$ and $v_{2}=(\mathrm{id} \otimes \bar{p}) v$. Assume that $v$ is a representation of $G$. Then $v_{1}$ and $v_{2}$ are representations of $G_{c}$,

and

$$
v=(\mathrm{id} \otimes \psi)\left(v_{1} \oplus v_{2}\right)
$$

$$
v_{2} \oplus v_{1}=\left(\mathrm{id} \otimes s_{c c}\right)\left(v_{1}(1) v_{2}\right) .
$$

Conversely if $v_{1}$ and $v_{2}$ are representations of $G_{c}$ acting on $K$ and satisfying relation (6.13) then $v$ introduced by $(6.12)$ is a representation of $G, v_{1}=(\mathrm{id} \otimes p) v$ and $v_{2}=(\mathrm{id} \otimes \bar{p}) v$. 
Remark. Relation (6.13) means that the smeared operators corresponding to $v_{1}$ commute with that of $v_{2}$ : for any $f_{1}, f_{2} \in \mathscr{A}_{c}^{\prime}$,

$$
\left[\left(\mathrm{id} \otimes f_{1}\right) v_{1},\left(\mathrm{id} \otimes f_{2}\right) v_{2}\right]=0 .
$$

Proof. We essentially repeat the proof of Theorem 4.4. If $v$ is a representation of $G$ then using (6.5) we see that $v_{1}$ and $v_{2}$ are representations of $G_{c}$. Moreover applying $(\mathrm{id} \otimes p \otimes \bar{p})$ to both sides of the relation

we get

$$
(\mathrm{id} \otimes \Phi) v=v(1) v
$$

$$
(\mathrm{id} \otimes(p \otimes \bar{p}) \circ \Phi) v=v_{1}\left(1 v_{2}\right.
$$

and (6.12) follows. In the similar way (6.13) is implied by (6.7).

Assume now that $v_{1}, v_{2} \in B(K) \otimes \mathscr{A}_{c}$ are representations of $G_{c}$ and that (6.13) holds. Applying (id $\otimes \Phi)$ to both sides of (6.12) we obtain (cf. (6.9)):

$$
\begin{aligned}
(\mathrm{id} \otimes \Phi) v & =(\mathrm{id} \otimes \psi \otimes \psi)\left(\mathrm{id} \otimes \mathrm{id}_{A_{c}} \otimes s_{c c} \otimes \mathrm{id}_{A_{c}}\right)\left(\mathrm{id} \otimes \Phi_{c} \otimes \Phi_{c}\right)\left(v_{1} \oplus v_{2}\right) \\
& =(\mathrm{id} \otimes \psi \otimes \psi)\left(\mathrm{id} \otimes \mathrm{id}_{A_{c}} \otimes s_{c c} \otimes \mathrm{id}_{A_{c}}\right)\left(v _ { 1 } \left(v_{1} \oplus v_{2}\left(v_{2}\right)\right.\right. \\
& =(\mathrm{id} \otimes \psi \otimes \psi)\left(v_{1} \unrhd v_{2} \oplus v_{1}\left(v_{2}\right)=v(v) .\right.
\end{aligned}
$$

In the next to the last step we used (6.13). It shows that $v$ is a representation of $G$. The remaining relations are obvious (cf. (6.10) and (6.11)). Q.E.D.

Relation (6.14) is obviously satisfied if one of the representations $v_{1}, v_{2}$ is trivial. For any $s=0,1 / 2,1, \ldots$ we consider representations $w^{s}$ and $\bar{w}^{s}$ of $G$ acting on $H^{s}$ such that

$$
\begin{array}{ll}
(\mathrm{id} \otimes p) w^{s}=u^{s}, & (\mathrm{id} \otimes \bar{p}) w^{s}=I_{B\left(H^{s}\right) \otimes \mathscr{A}_{c}}, \\
(\mathrm{id} \otimes p) \bar{w}^{s}=I_{B\left(H^{s}\right) \otimes \mathscr{A}_{c}}, & (\mathrm{id} \otimes \bar{p}) \bar{w}^{s}=u^{s} .
\end{array}
$$

According to (6.2), $w^{1 / 2}$ coincides with the representation $w$ introduced by (5.16). Similarly $\bar{w}^{1 / 2}$ coincides with $\bar{w}$.

In the following theorem " $\simeq$ " denotes the equivalence of representations.

\section{Theorem 6.3.}

$1^{\circ}$ For all $s, s^{\prime}=0,1 / 2,1,3 / 2, \ldots$

$$
w^{s} \odot \bar{w}^{s^{\prime}}
$$

are mutually non-equivalent, irreducible smooth representations of $S_{\mu} L(2, \mathbb{C})$.

$2^{\circ}$ Any smooth finite-dimensional representation $v$ of $S_{\mu} L(2, \mathbb{C})$ is equivalent to a direct sum of representations of the form (6.15).

$3^{\circ}$ For any $s, s^{\prime}=0,1 / 2,1,3 / 2, \ldots$,

$$
\begin{aligned}
& w^{s} \odot w^{s^{\prime}} \simeq \sum_{s^{\prime \prime}}^{\oplus} w^{s^{\prime \prime}}, \\
& \bar{w}^{s} \odot \bar{w}^{s^{\prime}} \simeq \sum_{s^{\prime \prime}}^{\oplus} \bar{w}^{s^{\prime \prime}},
\end{aligned}
$$

where the summation runs over $s^{\prime \prime}=\left|s-s^{\prime}\right|,\left|s-s^{\prime}\right|+1, \ldots, s+s^{\prime}$ and

$$
w^{s} \oplus \bar{w}^{s^{\prime}} \simeq \bar{w}^{s^{\prime}} \oplus w^{s} .
$$

Moreover $\bar{w}^{s}$ is the complex conjugate of $w^{s}$. 
Proof.

Ad $1^{\circ}$. Using (6.12) and taking into account (6.8) we see that matrix elements of $w^{s}$ ( $\bar{w}^{s^{\prime}}$ respectively) belong to $\mathscr{A}_{\text {hol }}\left(\mathscr{A}_{\text {hol }}^{*}\right.$ respectively). Therefore (cf. definitions (6.4))

$$
\begin{aligned}
& (\mathrm{id} \otimes p)\left(w^{s} \oplus \bar{w}^{s^{\prime}}\right)=u^{s} \odot I_{s^{\prime}}, \\
& (\mathrm{id} \otimes \bar{p})\left(w^{s} \oplus \bar{w}^{s^{\prime}}\right)=I_{s} \oplus u^{s^{\prime}},
\end{aligned}
$$

where $I_{r}=I_{B\left(H^{r}\right) \otimes A}\left(r=s, s^{\prime}\right)$ is the trivial representation of $S_{\mu} U(2)$ acting on $H^{r}$. According to Proposition 6.2 the above relations completely determine representation $w^{s} \oplus \bar{w}^{s^{\prime}}$.

Let $K$ be $a\left(w^{s} \odot \bar{w}^{s^{\prime}}\right)$-invariant subspace of $H^{s} \otimes H^{s^{\prime}}$. Then (cf. (6.19)) $K$ is $\left(u^{s} \odot I_{s^{\prime}}\right)$-invariant and $\left(u^{s}\right.$ is irreducible) $K=H^{s} \otimes L^{\prime}$, where $L^{\prime}$ is a subspace of $H^{s^{\prime}}$. Similarly using (6.20) we see that $K=L \otimes H^{s^{\prime}}$, where $L \subset H^{s}$. Therefore either $K=H^{s} \otimes H^{s^{\prime}}$ or $K=\{0\}$ and the irreducibility of $(6.15)$ follows.

Remark. Modifying in a suitable way definitions (6.4) and using the above method one can show that $\bar{w}^{s^{\prime}} \oplus w^{s}$ is also irreducible.

Assume now that $w^{s} \oplus \bar{w}^{s^{\prime}} \simeq w^{r} \oplus \bar{w}^{r^{\prime}}\left(s, s^{\prime}, r, r^{\prime}=0,1 / 2,1,3 / 2, \ldots\right)$. Then (cf. (6.19)) $u^{s} \oplus I_{s^{\prime}} \simeq u^{r} \oplus I_{r^{\prime}}$ and $s=r$. Similarly using (6.20) we obtain $s^{\prime}=r^{\prime}$. Statement $1^{\circ}$ is proved.

Ad $2^{\circ}$. Let $v$ be a smooth representation of $S_{\mu} L(2, \mathbb{C})$ acting on a finite-dimensional vector space $K$ and $v_{1}, v_{2}$ be the representations of $S_{\mu} U(2)$ introduced in Proposition 6.2. Representation $v_{1}$ can be decomposed into direct sum of factor representations (cf. Theorem 5.8 of [10]):

$$
\begin{aligned}
& K=\sum_{s}^{\oplus} H^{s} \otimes K^{s}, \\
& v_{1}=\sum_{s}^{\oplus} u^{s} \oplus I^{s},
\end{aligned}
$$

where $I^{s}=I_{B\left(K^{s}\right) \otimes A_{c}}$ is the trivial representation of $S_{\mu} U(2)$ acting on $K^{s}$. Taking into account (6.14) we see that $v_{2}$ is of the form

$$
v_{2}=\sum_{s}^{\oplus} I_{s} \uparrow v_{2 s}
$$

where for any $s, v_{2 s}$ is a representation of $S_{\mu} U(2)$ acting on $K^{s}$. Decomposing $v_{2 s}$ into a direct sum of irreducible representations and remembering that (6.19) and (6.20) determine $w^{s} \odot \bar{w}^{s^{\prime}}$ completely we obtain desired decomposition of $v$.

Ad $3^{\circ}$. According to (6.4) the restrictions of $p$ (and $\bar{p}$ ) to $\mathscr{A}_{\text {hol }}$ and $\mathscr{A}_{\text {hol }}^{*}$ are multiplicative. Therefore (6.16) and (6.17) follow from the similar decomposition for $u^{s} \oplus u^{s^{\prime}}$ (cf. Theorem 5.11 of [9]).

Using (6.16) ((6.17) respectively) one can easily check that $w^{s}\left(\bar{w}^{s}\right.$ respectively) is the only $(2 s+1)$-dimensional irreducible subrepresentation of $w^{\oplus(2 s)}\left(\bar{w}^{\oplus(2 s)}\right.$ respectively). Remembering that $\bar{w}$ is the complex conjugate of $w$ we conclude that $\bar{w}^{s}$ is the complex conjugate of $w^{s}$.

Similarly one can verify $(6.18): w^{s} \odot \bar{w}^{s^{\prime}}\left(\bar{w}^{s^{\prime}} \odot w^{s}\right.$ respectively) is the only $(2 s+1)$ $\left(2 s^{\prime}+1\right)$-dimensional irreducible subrepresentation of $w^{\oplus(2 s)} \oplus \bar{w}^{\oplus\left(2 s^{\prime}\right)}$ $\left(\bar{w}^{\oplus\left(2 s^{\prime}\right)} \oplus w^{\oplus(2 s)}\right.$ respectively). According to (1.5) $w^{\oplus(2 s)} \oplus \bar{w}^{\oplus\left(2 s^{\prime}\right)} \simeq \bar{w}^{\oplus\left(2 s^{\prime}\right)} \oplus w^{\oplus(2 s)}$ and (6.18) follows. Q.E.D. 
Let $\tau$ be the element of $M\left(A_{d}\right)$ such that

$$
\pi_{s}(\tau)=(-1)^{2 s} I_{s}
$$

for $s=0,1 / 2,1, \ldots$. Then $\tau$ belongs to the center of $M\left(A_{d}\right)$ and $I_{c} \otimes \tau$ commutes with the bicharacter $u$. Therefore (cf. (4.9))

$$
\sigma\left(I_{c} \otimes \tau\right)=\tau \otimes I_{c} .
$$

Let us notice (cf. (5.11)) that $\tau=f(a)$, where $f$ is the function defined on the set of all integer and half-integer powers of $\mu$ such that $f\left(\mu^{m}\right)=(-1)^{2 m}$. Clearly $f$ is multiplicative and using the first relation (5.4) we get

$$
\Phi_{d}(\tau)=\tau \otimes \tau
$$

It means that $\tau$ is a one-dimensional representation of $G_{d}$. Taking into account (6.22) and using Theorem 4.4 we see that $\tilde{\tau} \stackrel{\mathrm{df}}{=} I_{c} \otimes \tau$ is a one-dimensional representation of $S_{\mu} L(2, \mathbb{C})$. In particular

$$
\Phi(\tilde{\tau})=\tilde{\tau} \otimes \tilde{\tau} .
$$

This representation is not smooth: $\tilde{\tau} \notin \mathscr{A}$. Indeed according to Theorem $6.3 .2^{\circ}$ the trivial representation is the only smooth one-dimensional representation of $S_{\mu} L(2, \mathbb{C})$. We believe that

Conjecture 6.4. Any finite-dimensional representation $v$ of $S_{\mu} L(2, \mathbb{C})$ admits unique decomposition of the form:

$$
v=v_{1} \oplus\left(\tilde{\tau} \oplus v_{2}\right),
$$

where $v_{1}$ and $v_{2}$ are smooth.

To support this conjecture, one can easily show that the trivial representation and $\tilde{\tau}$ are the only one-dimensional representations of $S_{\mu} L(2, \mathbb{C})$ (use Theorem 4.4).

According to (6.21), $\tilde{\tau}$ is unitary and

$$
\operatorname{Sp} \tilde{\tau}=\{-1,1\}=\mathbb{Z}_{2} \text {. }
$$

Therefore the formula $\psi(x)=x(\tilde{\tau})$ introduces a monomorphism $\psi \in \operatorname{Mor}\left(C\left(\mathbb{Z}_{2}\right), A\right)$. Let $\Phi_{2}: C\left(\mathbb{Z}_{2}\right) \rightarrow C\left(\mathbb{Z}_{2}\right) \otimes C\left(\mathbb{Z}_{2}\right)$ be the comultiplication related to the group structure of $\mathbb{Z}_{2}$. Due to (6.23) the diagram

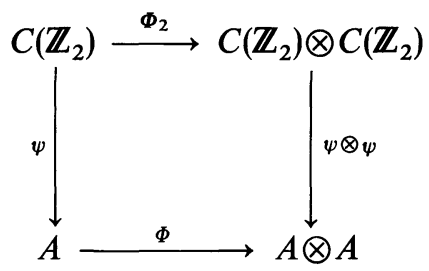

is commutative. It means that $\psi$ defines the quantum group epimorphism

$$
S_{\mu} L(2, \mathbb{C}) \rightarrow \mathbb{Z}_{2}
$$


The kernel of this epimorphism is a normal (quantum) subgroup of $S_{\mu} L(2, \mathbb{C})$ of index 2. It will be denoted by $S_{\mu} L^{+}(2, \mathbb{C})$. The algebra $A^{+}$of "all continuous vanishing at infinity functions on $S_{\mu} L^{+}(2, \mathbb{C})$ " is introduced by

$$
A^{+}=A / J \text {, }
$$

where $J$ is the closed two-sided ideal in $A$ generated by $\tilde{\tau}-I_{A}$. One can verify that

$$
J=A_{c} \otimes A_{d}^{-},
$$

where $A_{d}^{-}=\sum_{s=0}^{\infty} B\left(H^{s+1 / 2}\right) \subset A_{d}$. Therefore

$$
A^{+}=A_{c} \otimes A_{d}^{+},
$$
where $A_{d}^{+}=A_{d} / A_{d}^{-}$is isomorphic to $\sum_{s=0}^{\infty} B\left(H^{s}\right)$ (s runs over all nonnegative
integers).

Let $p \in \operatorname{Mor}(A, A / J)$ be the canonical epimorphism. The comultiplication related to $S_{\mu} L^{+}(2, \mathbb{C})$ is the only morphism $\Phi^{+} \in \operatorname{Mor}\left(A^{+}, A^{+} \otimes A^{+}\right)$such that the diagram

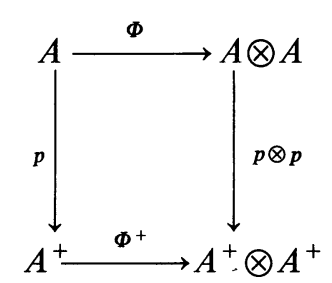

is commutative. The existence of $\Phi^{+}$follows easily from (6.23); the uniqueness is obvious. In the similar way one can define counit and coinverse related to $S_{\mu} L^{+}(2, \mathbb{C})$.

As in the classical case $S_{\mu} L(2, \mathbb{C})$ contains $\mathbb{Z}_{2}$ as a normal subgroup. The embedding $\mathbb{Z}_{2} \subset S_{\mu} L(2, \mathbb{C})$ is described by a morphism $\chi \in \operatorname{Mor}\left(A, C\left(\mathbb{Z}_{2}\right)\right)$ introduced by the formula $\chi=\chi^{\prime} \otimes e_{d}$, where $\chi^{\prime} \in \operatorname{Mor}\left(A_{c}, C\left(\mathbb{Z}_{2}\right)\right)$ is defined by

and

$$
\chi^{\prime}(\gamma)=0
$$

$$
\left(\chi^{\prime}(\alpha)\right)(t)=t
$$

for any $t \in \mathbb{Z}_{2}(t= \pm 1)$. One can easily check that the diagram

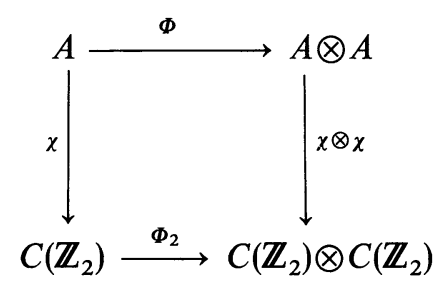

is commutative $\left(\mathbb{Z}_{2}\right.$ is a subgroup of $\left.S_{\mu} L(2, \mathbb{C})\right)$. Moreover for any $x \in A$,

$$
(\mathrm{id} \otimes \chi) \Phi(x)=x \otimes I_{C\left(\mathbb{Z}_{2}\right)} \Leftrightarrow(\chi \otimes \mathrm{id}) \Phi(x)=I_{C\left(\mathbb{Z}_{2}\right)} \otimes x
$$

(cf. Sect. 6 of [7]). This equivalence means that the subgroup $\mathbb{Z}_{2}$ is normal (any $\mathbb{Z}_{2}$-left-invariant "function on $S_{\mu} L(2, \mathbb{C})$ " is $\mathbb{Z}_{2}$-right-invariant). 
The quotient group $S_{\mu} L(2, \mathbb{C}) / \mathbb{Z}_{2}$ will be denoted by $S O_{\mu}(3,1)$. The algebra of "all continuous vanishing at infinity functions on $\mathrm{SO}_{\mu}(3,1)$ " is introduced by

$$
A^{\text {even }}=\left\{x \in A:(\mathrm{id} \otimes \chi) \Phi(x)=x \otimes I_{C\left(\mathbb{Z}_{2}\right)}\right\} .
$$

Clearly $A^{\text {even }}$ is a non-degenerate $C^{*}$-subalgebra of $A$ ("nondegenerate" means that the embedding $A^{\text {even }} \hookrightarrow A$ belongs to $\operatorname{Mor}\left(A^{+}, A\right)$ ). One can verify that

$$
A^{\text {even }}=A_{c}^{\text {even }} \otimes A_{d},
$$

where $A_{c}^{\text {even }}$ is the $C^{*}$-subalgebra of $A_{c}$ spanned by all even order monomials of $\alpha, \gamma$, $\alpha^{*}$, and $\gamma^{*}$. Due to (6.24), $\left.\Phi^{\text {even }} \stackrel{\text { df }}{=} \Phi\right|_{A^{\text {even }}}$ belongs to $\operatorname{Mor}\left(A^{\text {even }}, A^{\text {even }} \otimes A^{\text {even }}\right)$. $\Phi^{\text {even }}$ is the comultiplication related to $S_{\mu}(3,1)$. In a similar way one can introduce counit and coinverse related to this group.

One can easily check that $\tilde{\tau} \in M\left(A^{\text {even }}\right)$. Therefore one can repeat for $\mathrm{SO}_{\mu}(3,1)$ the procedure that produced $S_{\mu} L^{+}(2, \mathbb{C})$ out of $S_{\mu} L(2, \mathbb{C})$. On the other hand the normal subgroup $\mathbb{Z}_{2} \subset S_{\mu} L(2, \mathbb{C})$ is in fact contained in $S_{\mu} L^{+}(2, \mathbb{C})(\chi$ admits factorization $\chi=\chi^{+} \circ p$, where $\chi^{+} \in \operatorname{Mor}\left(A^{+}, C\left(\mathbb{Z}_{2}\right)\right)$. Therefore one can repeat for $S_{\mu} L^{+}(2, \mathbb{C})$ the procedure that produced $S O_{\mu}(3,1)$ out of $S_{\mu} L(2, \mathbb{C})$. In both cases we obtain the same quantum group denoted by $\mathrm{SO}_{\mu}^{+}(3,1)$. The algebra of "all continuous vanishing at infinity functions on $\mathrm{SO}_{\mu}^{+}(3,1)$ " is given by

$$
A^{\text {even+ }}=A_{c}^{\text {even }} \otimes A_{d}^{+} .
$$

One can easily check that $S_{\mu}^{+}(3,1)$ is the double group (cf. Sect. 4) built over $\mathrm{SO}_{\mu}(3)$.

Summarizing we obtain the following commutative diagram of quantum groups:

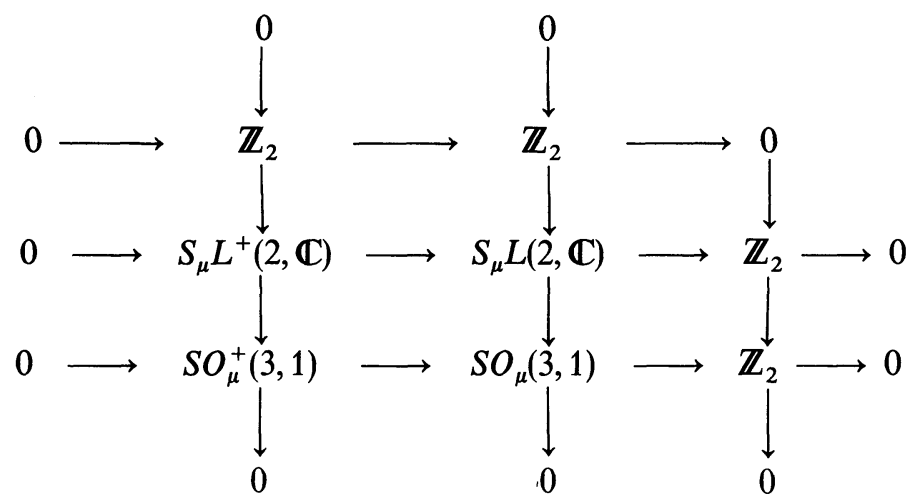

All rows and columns are exact.

Analysing the proof of Proposition 5.6 one can show that $J \cap \mathscr{A}=\{0\}$. Therefore the theory of smooth representations of $S_{\mu} L^{+}(2, \mathbb{C})$ is the same as that of $S_{\mu} L(2, \mathbb{C})$ : Theorem 6.3 with $S_{\mu} L(2, \mathbb{C})$ replaced by $S_{\mu} L^{+}(2, \mathbb{C})$ and $w^{s}, \bar{w}^{s^{\prime}}$ replaced by their restrictions to $S_{\mu} L^{+}(2, \mathbb{C})$ still holds. Moreover it seems (cf. Conjecture 6.4) that any finite-dimensional representation of $S_{\mu} L^{+}(2, \mathbb{C})$ is smooth $(\tilde{\tau}$ restricted to $S_{\mu} L^{+}(2, \mathbb{C})$ is trivial). As in the classical case $(6.15)$ is a representation of $S_{\mu}(3,1)$ (and $\left.\mathrm{SO}_{\mu}^{+}(3,1)\right)$ if and only if $s+s^{\prime}$ is integer. 
We would like to combine Theorem 6.3 with Theorem 4.6. A bicovariant $\mathscr{A}_{c}$-bimodule $\Gamma$ is called tame if the corresponding representation of $S_{\mu} L(2, \mathbb{C})$ is smooth. Let $\Gamma^{s r}(s, r=0,1 / 2,1,3 / 2, \ldots)$ be the bicovariant $\mathscr{A}_{c}$-bimodule corresponding to the representation $w^{s} \odot \bar{w}^{r}$. Taking into account Theorem 4.6 and 6.3 we obtain

\section{Theorem 6.5.}

$1^{\circ}$ The bimodules $\Gamma^{s r}(s, r=0,1 / 2,1,3 / 2, \ldots)$ are indecomposable. $\Gamma^{r s}$ is the complex conjugate of $\Gamma^{\text {sr }}$ and

$$
\Gamma^{s r} \otimes_{\mathscr{A}_{c}} \Gamma^{s^{\prime} r^{\prime}}=\sum_{s^{\prime \prime}, r^{\prime \prime}}^{\oplus} \Gamma^{s^{\prime \prime} r^{\prime \prime}}
$$

where the summation runs over $s^{\prime \prime}=\left|s-s^{\prime}\right|, \quad\left|s-s^{\prime}\right|+1, \ldots, s+s^{\prime}, \quad r^{\prime \prime}=\left|r-r^{\prime}\right|$, $\left|r-r^{\prime}\right|+1, \ldots, r+r^{\prime}$.

$2^{\circ}$ Any tame bicovariant $\mathscr{A}_{c}$-bimodule such that the space of left-invariant elements is finite-dimensional is a direct sum of bimodules of the form $\Gamma^{\text {sr }}$.

For the completeness we describe the structure of $\Gamma^{1 / 2,0}$. Let $\left(\omega_{1}, \omega_{2}\right)$ be the canonical basis in ${ }_{\text {inv }} \Gamma^{1 / 2,0}=\mathbb{C}^{2}$. Then any element $\omega \in \Gamma^{1 / 2,0}$ is of the form

$$
\omega=\omega_{1} a_{1}+\omega_{2} a_{2}
$$

where $a_{1}, a_{2} \in \mathscr{A}_{c}$ are uniquely determined and combining (5.2) with (4.35) we get

$$
\begin{aligned}
\alpha \omega_{1} & =\mu^{1 / 2} \omega_{1} \alpha, \\
\gamma \omega_{1} & =\mu^{1 / 2} \omega_{1} \gamma, \\
\alpha^{*} \omega_{1} & =\mu^{-1 / 2} \omega_{1} \alpha^{*}, \\
\gamma^{*} \omega_{1} & =\mu^{-1 / 2} \omega_{1} \gamma^{*}, \\
\alpha \omega_{2} & =\mu^{-1 / 2} \omega_{2} \alpha+\mu^{-1 / 2}\left(1-\mu^{2}\right) \omega_{1} \gamma^{*}, \\
\gamma \omega_{2} & =\mu^{-1 / 2} \omega_{2} \gamma-\mu^{-3 / 2}\left(1-\mu^{2}\right) \omega_{1} \alpha^{*}, \\
\alpha^{*} \omega_{2} & =\mu^{1 / 2} \omega_{2} \alpha^{*}, \\
\gamma^{*} \omega_{2} & =\mu^{1 / 2} \omega_{2} \gamma^{*} .
\end{aligned}
$$

Moreover denoting by ${ }_{\Gamma} \Phi$ the right action of $S_{\mu} U(2)$ on $\Gamma^{1 / 2,0}$ we have

$$
\begin{aligned}
& { }_{\Gamma} \Phi\left(\omega_{1}\right)=\omega_{1} \otimes \alpha_{c}+\omega_{2} \otimes \gamma_{c}, \\
& { }_{\Gamma} \Phi\left(\omega_{2}\right)=-\mu \omega_{1} \otimes \gamma^{*}+\omega_{2} \otimes \alpha^{*} .
\end{aligned}
$$

In [12], the $4 D_{+}$-differential calculus was introduced. Now we give an independent description of this calculus.

Let $\Gamma=\Gamma^{1 / 2,1 / 2}$ and $\Gamma^{\mu}$ be the external algebra built over $\Gamma$. We know that the restriction of $w \circlearrowleft \bar{w}$ to $S_{\mu} U(2)$ contains trivial subrepresentation. Therefore $\Gamma$ contains bi-invariant element. Denoting this element by $\tau$ we have

$$
d \theta=[\tau, \theta]_{\mathrm{grad}}=\left\{\begin{array}{ll}
\tau \wedge \theta-\theta \wedge \tau \\
\tau \wedge \theta+\theta \wedge \tau
\end{array} \text { if } \partial \theta \text { is } \begin{array}{ll}
\text { even } \\
\text { odd }
\end{array}\right.
$$

for any homogeneous element $\theta \in \Gamma^{\wedge}$ of grade $\partial \theta$. In particular

$$
d a=\tau a-a \tau
$$


for any $a \in \mathscr{A}_{c}$. One can check that

$$
\tau=\omega_{1} \otimes_{\mathscr{A}_{c}} \omega_{1}^{*}+\omega_{2} \otimes_{\mathscr{A}_{c}} \omega_{2}^{*},
$$

where $\omega_{1}$ and $\omega_{2}$ are elements of $\Gamma^{1 / 2,0}$ introduced above and $*$ denotes the canonical bimodule antihomomorphism $\Gamma^{1 / 2,0} \rightarrow \Gamma^{0,1 / 2}$.

\section{A. Appendix}

In the paper we deal with linear mappings acting between tensor spaces built over "elementary" vector spaces $A_{c}$ and $A_{d}$. In most cases the mappings are introduced as compositions of tensor products of "elementary" mappings such as $m_{c}, m_{d}, \Phi_{c}$, $\Phi_{d}, e_{c}, e_{d}, \kappa_{c}, \kappa_{d}, h_{c}, h_{d L}, h_{d R}, \sigma, \ldots$. The traditional tensor notation becomes illegible if the number of elementary mappings entering a single formula is large. Dealing with such cases it is convenient to use the diagram notation that generalizes the one introduced in [11]. We recall that in this notation mappings are represented by diagrams consisting of lines of approximately vertical direction and vertices. Lines represent identity mappings acting on the elementary vector spaces. To make our diagrams more readable we shall use "coloured" lines associating a definite colour with each elementary vector space. In what follows continuous (for $A_{c}$ ) and dashed (for $A_{d}$ ) lines will be used.

The line is called incoming (outgoing respectively) if it has a free upper (lower respectively) end placed on the horizontal line that bounds the diagram from above (below respectively). Each line either is incoming (outgoing respectively) or starts from (ends up at respectively) a vertex. Lines must not intersect.

Diagrams with $N$ incoming and $M$ outgoing lines represent linear mappings acting from $A_{1} \otimes A_{2} \otimes \ldots \otimes A_{N}$ into $A_{1}^{\prime} \otimes A_{2}^{\prime} \otimes \ldots \otimes A_{M}^{\prime}$ (where $A_{1}, A_{2}, \ldots, A_{N}$ $\left(A_{1}^{\prime}, A_{2}^{\prime}, \ldots, A_{M}^{\prime}\right.$ respectively) are elementary vector spaces corresponding to the colours of incoming (outgoing respectively) lines). If $M=0$ (no outgoing line) then the diagram represents a mapping into $\mathbb{C}$, i.e. a linear functional defined on $A_{1} \otimes A_{2} \otimes \ldots \otimes A_{N}$. Similarly if $N=0$ (no incoming line) then the diagram represents a mapping from $\mathbb{C}$, i.e. an element of $A_{1}^{\prime} \otimes A_{2}^{\prime} \otimes \ldots \otimes A_{M}^{\prime}$. If $M=N=0$ (no external line) then the diagram represents a complex number.

The mapping represented by a diagram $\alpha$ will be denoted by $[\alpha]$. The diagram representing the tensor product $[\alpha] \otimes[\beta]$ is obtained by drawing $\alpha$ side by side $\beta(\alpha$ on the left). Let us notice that the composition $[\alpha] \circ[\beta]$ is well defined only if the number and colour composition of outgoing lines of $\beta$ coincide with the number and colour composition of incoming lines of $\alpha$. The diagram representing $[\alpha] \circ[\beta]$ is obtained by placing the diagram $\alpha$ below the diagram $\beta$ and connecting the lower ends of $\beta$ with the corresponding upper ends of $\alpha$.

Each "elementary" mapping will be represented by a single vertex diagram. It consists of a small circle (containing inside the symbol denoting the considered mapping) and suitable incoming and outgoing lines attached to it. To simplify the diagrams we shall use
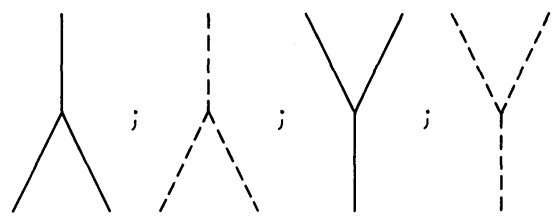

and

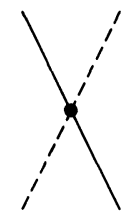


instead of

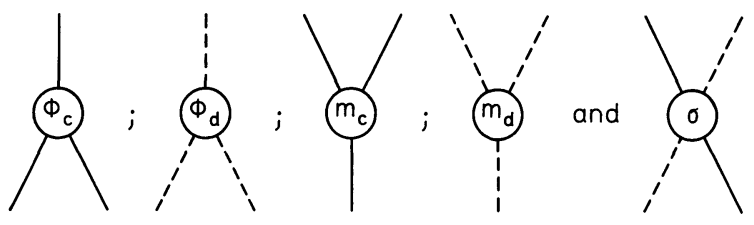

respectively. The flip automorphisms: $s_{c c}: A_{c} \otimes A_{c} \rightarrow A_{c} \otimes A_{c}, s_{c d}: A_{c} \otimes A_{d} \rightarrow A_{d} \otimes A_{c}$, $s_{d c}: A_{d} \otimes A_{c} \rightarrow A_{c} \otimes A_{d}$ and $s_{d d}: A_{d} \otimes A_{d} \rightarrow A_{d} \otimes A_{d}$ will be represented by diagrams

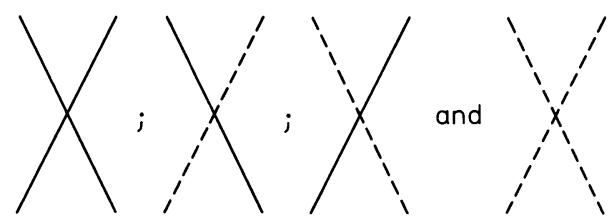

respectively. Each of the above diagrams consists of four lines and one vertex (the lines do not intersect!). The mappings $\mathbb{C} \ni \lambda \rightarrow \lambda I_{c} \in A_{c}$ and $\mathbb{C} \ni \lambda \rightarrow \lambda I_{d} \eta A_{d}$ will be represented by diagrams

respectively.

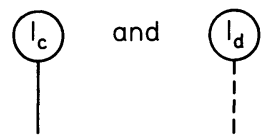

In the diagram notation, relations (3.4), (3.6), and (3.11)-(3.12) mean that

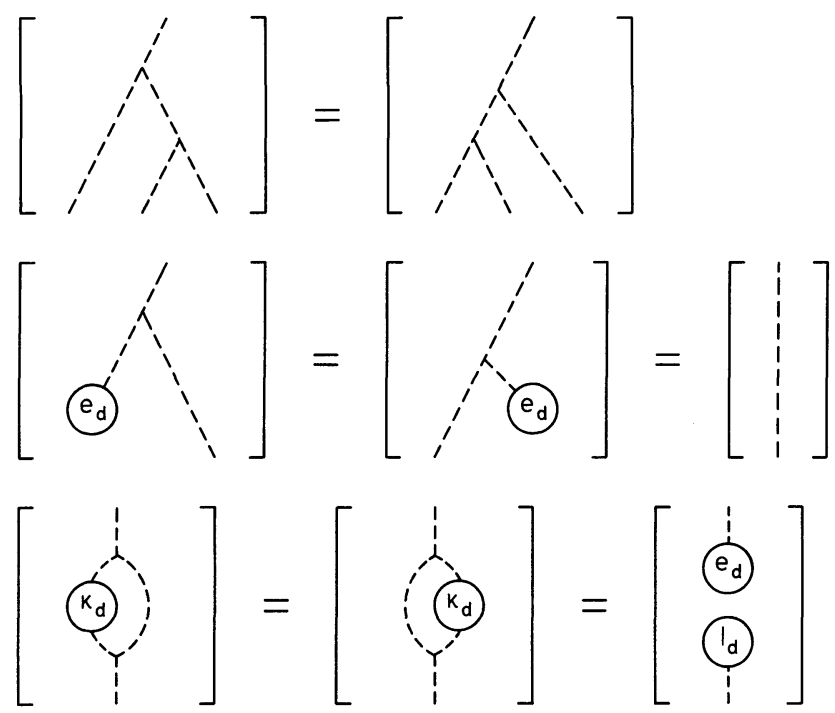

Similarly the relations (3.22) and (3.25) can be rewritten in the following form

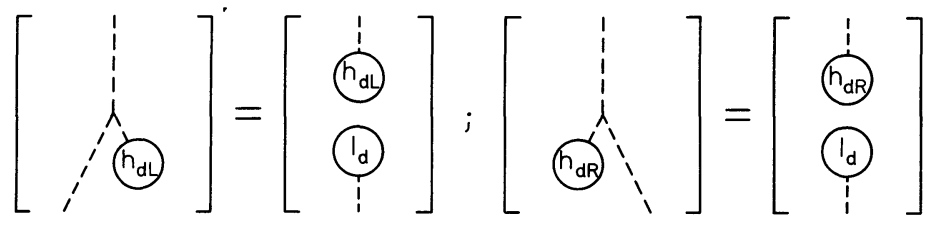


Replacing in the above diagrams $e_{d}, \kappa_{d}, I_{d}$ by $e_{c}, \kappa_{c}, I_{c}$ respectively, $h_{d L}$ and $h_{d R}$ by $h_{c}$ and drawing continuous lines instead of dashed ones we obtain the corresponding relations for $G_{c}$. We number these relations from (A.5) to (A.8). The relations (4.10)-(4.15) describing the properties of $\sigma$ have the following form:
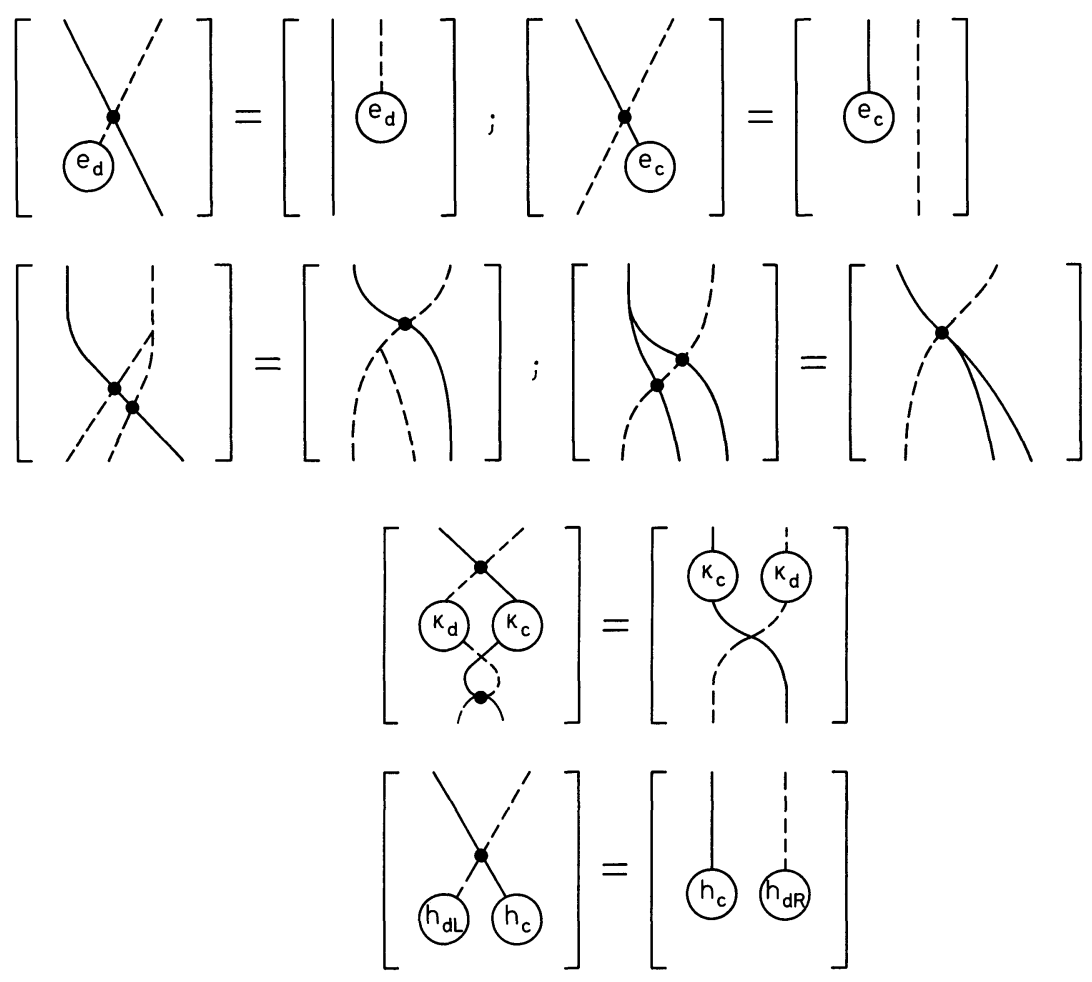

The basic notions related to the double group $G$ are introduced in the following way (cf. (4.16)-(4.18)):

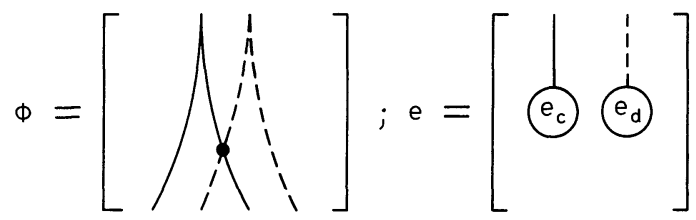

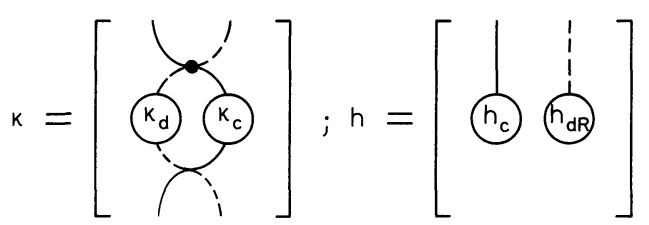


Now we can easily prove the relations (4.19)-(4.23) of Theorem 4.1. Using(A.1), (A.5), and (A.10) we have

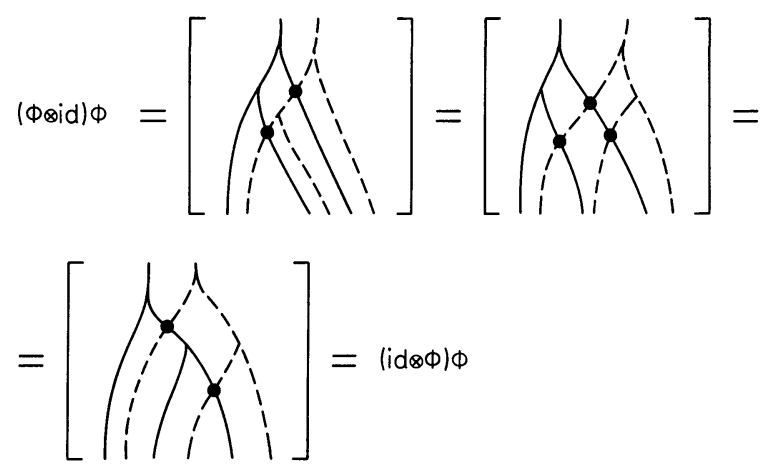

and (4.19) follows. Similarly using (A.9), (A.2), and (A.6) we get

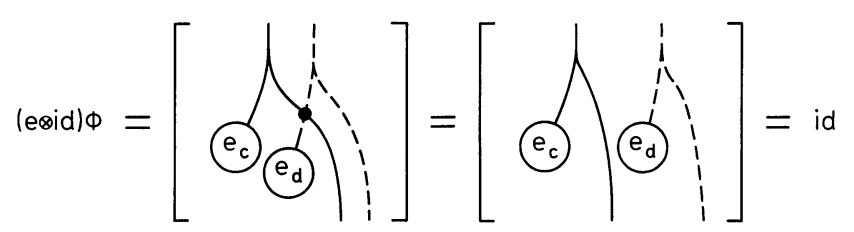

and in the same manner one can verify the second part of (4.20). Using (A.10), (A.7), (A.9), and (A.3) we compute

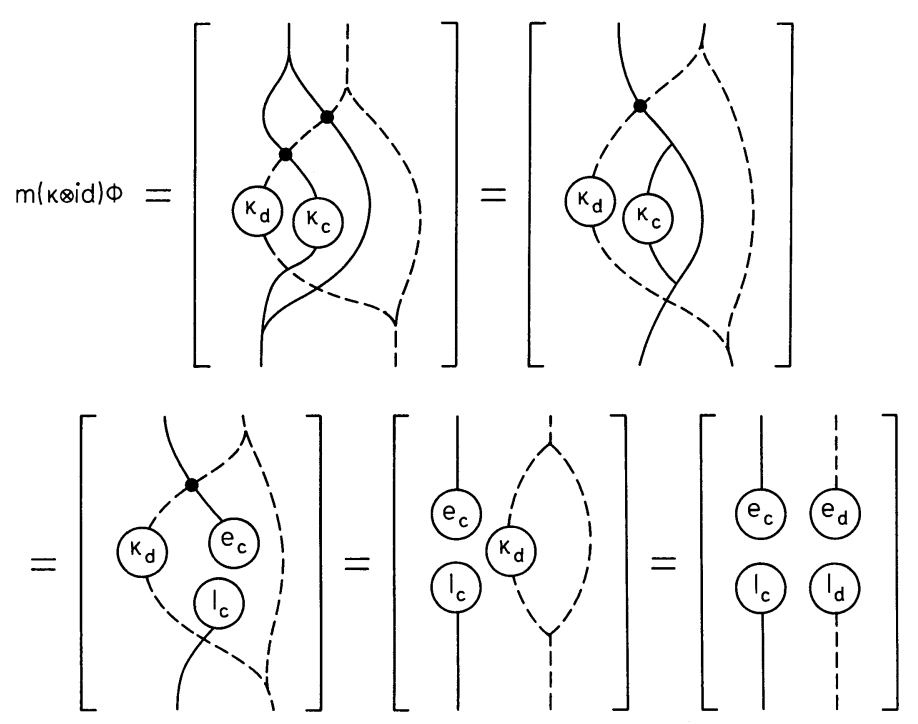

and (4.22) follows. In the same manner one can verify (4.23). Relation (4.21) easily follows from definition of $\kappa$, (A.11), (3.9) and an analogous formula for $\kappa_{c}$. Finally we show that the measure $h$ is left and right invariant. Using (A.12), (A.10), (A.8) 
and once more (A.12) we get

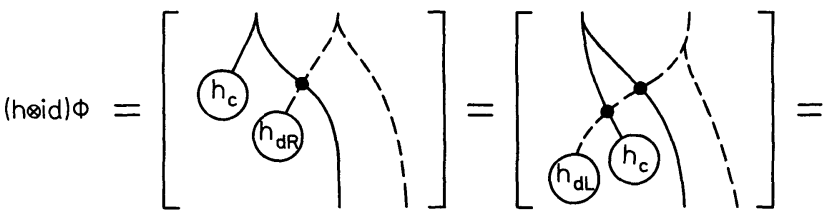

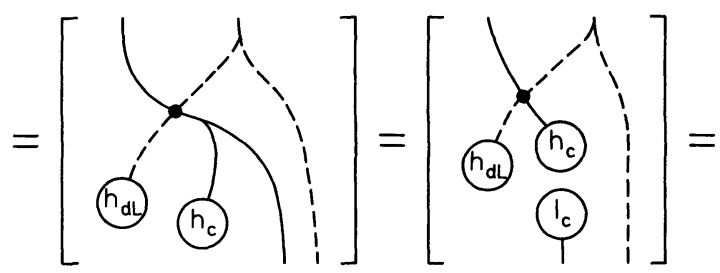

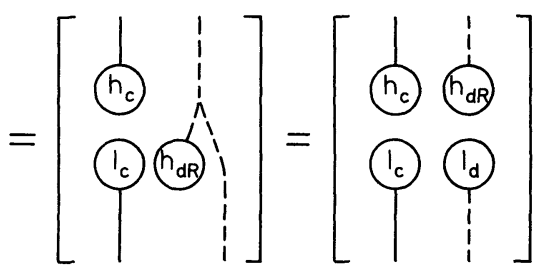

Similarly one can verify the second relation of Theorem 4.2:

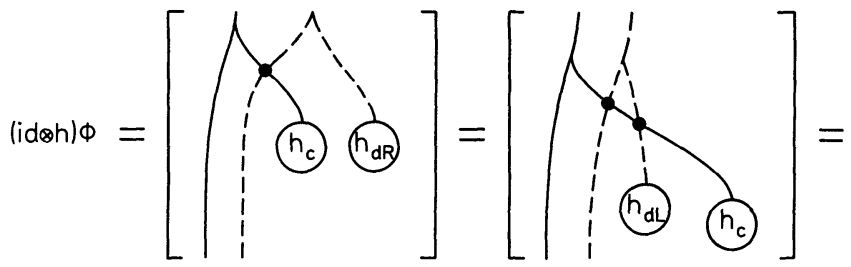

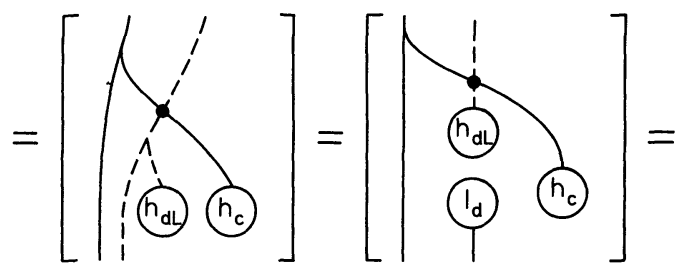

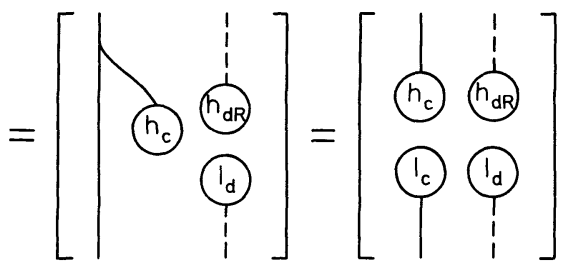


Acknowledgements. We are very indebted to Prof. Alain Connes for the remark that was the starting point of the research presented in this paper. The main results were obtained during our visit to the Mittag Leffler Institute in March and April 1989. We are grateful to Prof. E. Størmer and Prof. D. Laksov for their kind hospitality creating the perfect atmosphere for the work. We also thank the Swedish Royal Academy of Sciences for the financial support. The financial support was also provided by Polish Ministry of National Education (Grant I.10). The authors are grateful to Mrs. M. Lapko for preparing the pictures.

\section{References}

1. Abe, E.: Hopf algebras, Cambridge Tracts in Mathematics, vol. 74. Cambridge: Cambridge Univ. Press 1977

2. Connes, A.: Non-commutative differential gometry. Institut des Hautes Etudes Scientifiques. Extrait des Publications Mathématiques No. 62 (1986)

3. Drinfeld, V.G.: Quantum groups. Proceedings of the International Congress of Mathematicians. Berkeley, CA, USA (1986), pp. 793-820

4. Kruszyński, P., Woronowicz, S.L.: A noncommutative Gelfand Naimark theorem. J. Operator Theory 8, 361-389 (1982)

5. Manin, Yu.I.: Quantum groups and noncommutative geometry. Centre de Recherche Mathematique, Montreal 1988

6. Phillips, N.C.: Inverse limits of $C^{*}$-algebras and applications. Operator algebras and application, Vol.1. London Mathematical Society, Lecture Note Series, vol. 135, pp. 127-186. Cambridge: Cambridge University Press 1988

7. Podleś, P.: Quantum spheres. Lett. Math. Phys. 14, 193-202 (1987)

8. Woronowicz, S.L.: Pseudospaces, pseudogroups and Pontryagin duality. Proceedings of the International Conference on Mathematics and Physics, Lausanne 1979. Lecture Notes in Physics, vol. 116, pp. 407-412. Berlin, Heidelberg, New York: Springer 1980

9. Woronowicz, S.L.: Twisted $S U(2)$ group. An example of a noncommutative differential calculus. Publ. RIMS, Kyoto University 23, 117-181 (1987)

10. Woronowicz, S.L.: Compact matrix pseudogroups. Commun. Math. Phys. 111, 613-665 (1987)

11. Woronowicz, S.L.: Tannaka - Krein duality for compact matrix pseudogroups. Twisted $S U(N)$ groups. Invent. Math. 93, 35-76 (1988)

12. Woronowicz, S.L.: Differential calculus on compact matrix pseudogroups (quantum groups). Commun. Math. Phys. 122, 125-170 (1989)

13. Woronowicz, S.L.: Elements affiliated with a $C^{*}$-algebra and non-compact quantum groups (to appear)

Communicated by A. Connes

Received October 25, 1989 
